\title{
Transitional shock-wave/boundary-layer interactions in hypersonic flow
}

\author{
N. D. Sandham ${ }^{1, \dagger}$, E. Schülein ${ }^{2}$, A. Wagner ${ }^{2}$, S. Willems ${ }^{3}$ and J. Steelant ${ }^{4}$ \\ ${ }^{1}$ Aerodynamics and Flight Mechanics Group, University of Southampton, Southampton SO17 1BJ, UK \\ ${ }^{2}$ German Aerospace Centre (DLR), Institute of Aerodynamics and Flow Technology, Bunsenstrasse 10, \\ Göttingen, 37073, Germany \\ ${ }^{3}$ German Aerospace Centre (DLR), Institute of Aerodynamics and Flow Technology, Linder Höhe, \\ 51147 Köln, Germany \\ ${ }^{4}$ European Space Research and Technology Centre, Propulsion Design and Aerothermodynamics \\ Section, Keplerlaan 1, 2200 AG Noordwijk, The Netherlands
}

(Received 23 December 2013; revised 6 May 2014; accepted 6 June 2014)

Strong interactions of shock waves with boundary layers lead to flow separations and enhanced heat transfer rates. When the approaching boundary layer is hypersonic and transitional the problem is particularly challenging and more reliable data is required in order to assess changes in the flow and the surface heat transfer, and to develop simplified models. The present contribution compares results for transitional interactions on a flat plate at Mach 6 from three different experimental facilities using the same instrumented plate insert. The facilities consist of a Ludwieg tube (RWG), an open-jet wind tunnel $(\mathrm{H} 2 \mathrm{~K})$ and a high-enthalpy free-piston-driven reflected shock tunnel (HEG). The experimental measurements include shadowgraph and infrared thermography as well as heat transfer and pressure sensors. Direct numerical simulations (DNS) are carried out to compare with selected experimental flow conditions. The combined approach allows an assessment of the effects of unit Reynolds number, disturbance amplitude, shock impingement location and wall cooling. Measures of intermittency are proposed based on wall heat flux, allowing the peak Stanton number in the reattachment regime to be mapped over a range of intermittency states of the approaching boundary layer, with higher overshoots found for transitional interactions compared with fully turbulent interactions. The transition process is found to develop from second (Mack) mode instabilities superimposed on streamwise streaks.

Key words: high-speed flow, shock waves, transition to turbulence

\section{Introduction}

Shock-wave/boundary-layer interactions (SWBLI) are of considerable interest technologically, due to their potential to cause flow separation, unsteadiness, high local surface heating and possible engine intake unstart, and also from a more fundamental

$†$ Email address for correspondence: n.sandham@soton.ac.uk 
perspective, since the flow phenomena occurring in strong interactions are not fully understood and conventional modelling approaches are known to be problematic. Reviews of SWBLI including the modelling issues may be found in Delery (1985), Dolling (2001) and Babinsky \& Harvey (2011). Much of the previous work has been for fully turbulent interactions, however transitional interactions are common in wind tunnel and small flight vehicle testing and are also relevant to drag reduction techniques where laminar flow is maintained up to a shock. The present investigation, combining experimental and simulation approaches, was motivated by the desire to provide a better fundamental understanding of transitional SWBLI and the need for reference data from large-scale facilities. The nominal Mach number for the study is 6, which places the work in the context of hypersonic flight, but remaining in the continuum flow regime without the need to consider rarefied flow or real gas effects. The configuration chosen for study is a flat plate with shock impingement, which is geometrically simple and has many similarities to the alternative ramp arrangement.

Transitional SWBLI are expected to contain features of both fully laminar and fully turbulent interactions. Laminar boundary layers are less able to withstand the large adverse pressure gradient that arises when a shock wave impinges on a boundary layer. The recirculation bubble that results from the interaction is well known, starting from the experimental study of Hakkinen et al. (1959). Near the separation point it is possible to carry out a triple deck analysis (Stewartson \& Williams 1969), which was shown by Katzer (1989) to be in good agreement with two-dimensional numerical simulations. Turbulent flow interactions are less well understood. In particular, modelling based on the Reynolds-averaged Navier-Stokes equations is severely challenged, due to the need to represent attached and detached shear layers, with the outer edge of the boundary layer encountering strong shocks and expansion waves, as well as the processes of separation and reattachment, which are already well known as weaknesses of RANS approaches in low-speed flow. In addition, turbulent interactions are known to feature additional low-frequency modes of unsteadiness (see, for example, Piponniau et al. (2009) and Touber \& Sandham (2011) for recent investigations into this phenomenon).

Linear stability analysis of laminar flows provides some insight into the transition processes, particularly for configurations with low background noise levels. For flat plate boundary layers developing under free streams with Mach numbers above $~ 3$ (depending on wall temperature) the so-called second (Mack) modes (Mack 1984) are most unstable, while the first mode instability occurs in the form of oblique waves. Second (Mack) modes have been observed experimentally on cones, see for example Kendall (1975), Laurence et al. (2012) and Wagner et al. (2013). In separation bubbles the situation is more complicated since the most rapid wave growth occurs in detached shear layers. The relevance of linear mechanisms has been studied, for example in Pagella, Babucke \& Rist (2004), showing good agreement with direct numerical simulations (DNS) and also a close correspondence between ramp flow and flow with shock impingement. For the shock impingement problem Yao et al. (2007) presented simulations of strong SWBLI at $M=2,4.5$ and 6.85, finding significantly reduced growth factors at the higher Mach numbers. Interestingly the main transition mechanism was found to be due to oblique modes. The most unstable modes from linear analysis were also present in the DNS, however differences emerged with increasing Mach number. Linear theory has also been used to study the global stability characteristics of shock-induced separation bubbles. For example, Robinet (2007) attributed the emergence of 3D time-dependent solutions in laminar SWBLI to the presence of a global mode of instability. 
The transition process initiated with linear instabilities is only one of several routes through to turbulence, for which Reshotko (2001) provided a revised version of Morkovin's road map including transient growth approaches to bypass transition. DNS of bypass transition at low speed have been shown, for example by Jacobs \& Durbin (2001), to include turbulent spots. Similar spots have been observed previously in high-speed flows (Fiala et al. 2006) and their growth rates measured in DNS (Redford, Sandham \& Roberts 2012). For transitional flows, particularly those with higher background disturbances, the range of phenomena between fully laminar and fully turbulent interactions may be described using the idea of boundary-layer intermittency, introduced by Narasimha (1985), based on the idea of the transition process being dominated by the growth and merging of turbulent spots. An intermittency factor $\gamma$ is introduced that can give a measure of the state of the boundary layer at a point of shock interaction, equal to zero in laminar flow and one in turbulent flow. There are multiple definitions of intermittency that can be employed based on what can be easily measured (Hedley \& Keffer 1974). Common definitions involve the application of a threshold to a particular quantity, usually sampled over a window in time or space. Where the threshold is exceeded, turbulent flow is assumed, otherwise the flow is assumed to be laminar. An alternative approach was proposed by Schneider (1995) based on plots of the probability density function (p.d.f.) of a relevant quantity, for example the skin friction. For his experiments, Schneider found two peaks in the p.d.f. that could be attributed to the laminar and turbulent regions of flow (outside and inside turbulent spots, respectively), leading to a method of defining intermittency without an explicit threshold. In addition to its use in quantifying the transition process, intermittency has been found to be an effective parameter in the development of transition models (see, for example, Steelant \& Dick 1996; Langtry \& Menter 2009; Steelant \& Dick 2001).

Hypersonic facilities represent particular challenges for experimental investigations of transition. Many facilities have very short run times, limiting the data that can be extracted. In addition, the environment for instrumentation is severe, with high levels of heating and possible diaphragm debris, which may occur as a result of the diaphragm rupture process. The environment is often of high background noise level, mainly due to sound radiation from turbulent boundary layers on nozzle walls, although some facilities have been designed to be quiet by the use of boundary-layer bleeds (see Schneider 2008 for a review). A study of transition with SWBLI was presented by Benay et al. (2006) for a hollow cylinder flare model in the ONERA $\mathrm{R} 2 \mathrm{Ch}$ wind tunnel at Mach 5. The stagnation pressure was varied to provide cases that were fully laminar, transitional and fully turbulent. The flow was found to be characterised by well-organised Görtler vortices, however these were not directly implicated in the transition process. The experimental study was complemented by laminar and turbulent flow calculations (using turbulence models for the latter) and a linear stability analysis based on laminar base flows. Laminar cases were characterised by multiple steady recirculation regions between the initial separation and reattachment. Convective instabilities were found to be largest for oblique first modes (in agreement with the study of Yao et al. 2007 cited above). A continuation of this work was published by Bur \& Chanetz (2009). This study noted that heat transfer can be higher in transitional interactions. Although the natural transition cases did not extend as far as fully turbulent interaction, trips were used so that the fully turbulent cases could be studied. The highest heat flux was found under cases of natural transition when Görtler vortices were present.

The objective of the present study is to develop an understanding of the effect of the intermittency of the boundary layer on the shock interaction. In particular, 
measurements will be presented of the heat transfer to the wall in large-scale facilities that are rarely used for fundamental studies of laminar-turbulent transition. For turbulent interactions the peak Stanton number downstream of flow reattachment is many times the heat transfer of the undisturbed boundary layer and transitional interactions often lead to an overshoot in skin friction and heat transfer. In the present contribution, an instrumented test plate was manufactured and tests were run in three facilities, complemented by DNS where feasible (note that, due to the cold wall conditions and consequent low values of viscosity close to the wall, the HEG case would have required computational resources far in excess of those available to resolve the details of the turbulent flow). In the following $\S 2$ the experimental and computational set-ups are described. In $\S 3$ the mean flow pattern is described and comparisons are made between experiments and simulations. Sections 4 and 5 consider the intermittency and the transition process, respectively.

\section{Experiments and numerical simulations}

\subsection{Experimental facilities}

The present study employed three experimental facilities, the first of which is a Ludwieg tube known as the RWG (Rohrwindkanal, Göttingen). The facility covers a Mach number range from 2 to 6.85. After opening the gate valve air flow is started by expansion waves, which travel to the closed end of the pressurised storage tube and reflect back. Until these waves reach the nozzle throat, the test gas flows out at nearly constant stagnation conditions through the nozzle and test section (with diameter $0.5 \mathrm{~m}$ ) to the dump tank. At the nominal Mach 6 condition used here the stagnation pressure ranges from 0.4 to $3.4 \mathrm{MPa}$, the stagnation temperature from 410 to $640 \mathrm{~K}$ and the unit Reynolds number $R e_{1}=\rho_{\infty} U_{\infty} / \mu_{\infty}\left(\mathrm{m}^{-1}\right)$ can vary from $2 \times 10^{6} \mathrm{~m}^{-1}$ to $28 \times 10^{6} \mathrm{~m}^{-1}$. In the context of the present study the RWG provides a convenient facility to sweep through a wide range of unit Reynolds numbers. Runs were made covering a range of cases with and without shock impingement $\left(4^{\circ}\right.$ wedge with inviscid shock impingement nominally $337 \mathrm{~mm}$ from the leading edge) for unit Reynolds numbers ranging from 1.7 to $12.7 \times 10^{6} \mathrm{~m}^{-1}$. The Mach number was $5.98 \pm 0.06$. The experiments in RWG were carried out in two phases, one (denoted phase 1 or RWG1) covering a series of experiments at different values of $R e_{1}$ and the other (denoted as phase 2 or RWG2) covering a series of cases with different shock impingement locations chosen for comparison with DNS. For the second phase the leading edge of the plate was polished to be sharper (leading-edge radius of the order of $10 \mu \mathrm{m}$ ) than in the first phase, with some interesting consequences on the results. During phase 2 of the work free-stream Pitot-pressure disturbance levels were measured using a fast-response pressure transducer positioned in the flow such that the opening was facing the direction of flow. The root-mean-square disturbance levels were observed to decrease monotonically from $2.22 \%$ at $R e_{1}=1.9 \times 10^{6} \mathrm{~m}^{-1}$ to $1.14 \%$ at $R e_{1}=17.5 \times 10^{6} \mathrm{~m}^{-1}$, with interpolated values of $1.62 \%$ and $1.45 \%$ at the two main run conditions of $R e_{1}=4 \times 10^{6} \mathrm{~m}^{-1}$ and $R e_{1}=6 \times 10^{6} \mathrm{~m}^{-1}$, respectively.

The second facility is the $\mathrm{H} 2 \mathrm{~K}$ (Hypersonic wind tunnel Cologne), which is a blowdown wind tunnel with a free-jet test section exhausting to a vacuum chamber. A test time of $30 \mathrm{~s}$ is obtained. The test gas can be heated with resistance heaters up to $1100 \mathrm{~K}$. A contoured Mach 6 nozzle with an exit diameter of $600 \mathrm{~mm}$ was used for the present experiments, which were run for nominal unit Reynolds numbers of 3 , 6 and $12 \times 10^{6} \mathrm{~m}^{-1}$ for cases with and without a shock impingement, with the shock generated by a $4^{\circ}$ wedge. For the cases with shock impingement two impingement 
locations were used, located 239 and $331 \mathrm{~mm}$ from the leading edge. The range of unit Reynolds number was similar in $\mathrm{H} 2 \mathrm{~K}$ and RWG, allowing a comparison of transition to turbulence in two different facilities.

The third facility is the HEG (High Enthalpy Shock Tunnel, Göttingen), which was originally designed to investigate high-temperature effects on the aerothermodynamics of entry and re-entry space vehicles, but has since had its operational range extended to cover low-altitude Mach 6 conditions up to Mach 10 at $33 \mathrm{~km}$ altitude. The HEG facility consists of a secondary reservoir and compression tube, separated from the adjoining shock tube by the primary diaphragm, with the nozzle and test section downstream. The high-pressure air in the secondary reservoir drives the piston down the compression tube. In the present experiments a pressure of $4 \mathrm{MPa}$ is used to accelerate the $850 \mathrm{~kg}$ piston down the $33 \mathrm{~m}$ long compression tube with an inner diameter of $550 \mathrm{~mm}$. When the $43 \mathrm{MPa}$ burst pressure of the $8 \mathrm{~mm}$ thick stainless steel diaphragm is reached the driver gas, consisting of a helium/argon mixture is heated to $4000 \mathrm{~K}$. The resulting strong shock wave travels down the $17 \mathrm{~m}$ shock tube and reflects off the end wall, heating the gas to the required reservoir conditions. Subsequently the test gas expands through a convergent-divergent nozzle, with an exit diameter of $0.43 \mathrm{~m}$, into the test section. At the selected flow condition the Mach number was 5.7, the unit Reynolds number varied between 13.8 and $14.5 \times 10^{6} \mathrm{~m}^{-1}$ and the free-stream temperature between 409 and $422 \mathrm{~K}$. This flow condition provides a contrast in the ratio of wall temperature to stagnation temperature of 0.11 in $\mathrm{HEG}$, compared with $0.49-0.59$ in RWG and $\mathrm{H} 2 \mathrm{~K}$ for stagnation temperatures in the range $500-600 \mathrm{~K}$. For the typical run conditions presented here, the ratio of wall temperature to free-stream temperature was approximately 0.7 for HEG compared with 4.5 and 4.7 for RWG and $\mathrm{H} 2 \mathrm{~K}$, respectively. The test condition chosen for HEG in the present paper has a total temperature of approximately $2680 \mathrm{~K}$ in the nozzle reservoir, while the wind tunnel model is at room temperature before each test and a surface temperature increase in the order of $O(10 \mathrm{~K})$ is observed. Thus, the boundary layer is highly cooled. A similar test condition on a slender 7 half-angle cone at Mach 7.4 revealed a maximum temperature of around $800 \mathrm{~K}$ in the boundary layer. Although vibrational excitation of oxygen is expected in that temperature range, the effect on the boundary-layer transition process is expected to be negligible. The HEG flow conditions were calculated using the DLR TAU code assuming the gas to be in equilibrium. The assumption is supported by wind tunnel calibrations which were reported in Hannemann, Martinez Schramm \& Karl (2008).

\subsection{Measurement techniques}

The basic configuration for the wind tunnel model is shown in figure 1. In RWG and $\mathrm{H} 2 \mathrm{~K}$ tests the leading edge was polished sharp whereas in HEG the leading edge was given a radius of curvature of $0.16 \mathrm{~mm}$ to get natural transition to occur at a convenient location on the plate. The shock generator plate is $210 \mathrm{~mm}$ long and $300 \mathrm{~mm}$ wide and is mounted with a deflection angle of $4^{\circ}$ in the RWG and H2K tests and both 2 and $4^{\circ}$ in HEG. The model is provided with inserts for different instrumentation. One insert is for infrared thermography and includes 10 equally spaced coaxial thermocouples (Type E). The other two are labelled $\mathrm{T}$ and $\mathrm{P}$ and are shown in figure 2. Insert $T$ is provided with 17 thin film gauges, 24 coaxial thermocouples (Type E) and 14 Kulite $^{(\mathrm{R})}$ pressure transducers, whereas insert $\mathrm{P}$ is provided with five coaxial thermocouples (Type E), six $\mathrm{PCB}^{(\mathrm{R})}$ pressure sensors (five active, one blind, frequency range $11-1000 \mathrm{kHz}$ ) and an atomic layer thermopile 

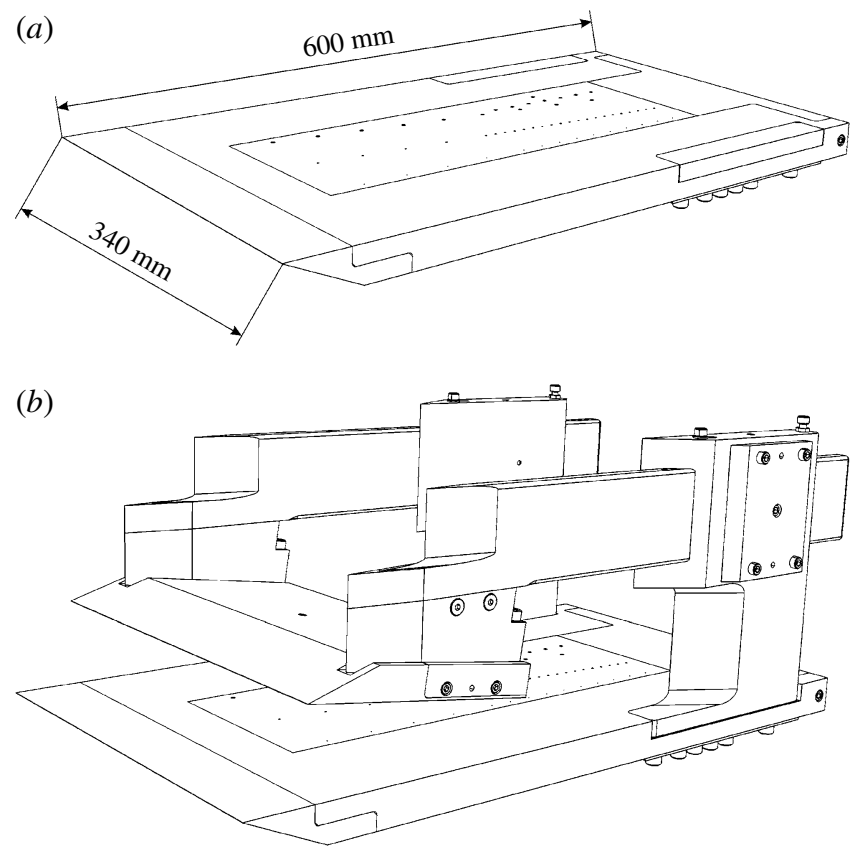

FIGURE 1. Schematic of the wind tunnel model showing configurations: $(a)$ without the shock generator, showing the leading edge and plate insert and $(b)$ as mounted with the shock generator. When the shock generator is not present the gap at the rear of the test plate is filled.

(ALTP) heat flux sensor (frequency range $17 \mathrm{~Hz}-1 \mathrm{MHz}$ ) that can be used at five different locations as shown on the figure. In the following paragraphs we comment in more detail on the various measurement techniques.

In RWG and $\mathrm{H} 2 \mathrm{~K}$, quantitative infrared thermography (QIRT) is used as a reference technique to detect laminar-turbulent transition in the boundary layer and quantitatively to measure the heat flux distribution on the model surface. To determine the heat transfer rate a thick-walled model fabricated from a material with low thermal conductivity is used. The heat transfer rate can then be calculated using an assumption of a semi-infinite wall, using the time history of the surface temperature acquired during the wind tunnel run. In the present study the insert was fabricated from PEEK and the time history of surface temperature was recorded using a high-resolution infrared camera at a frame rate of $105 \mathrm{~Hz}$ and an exposure time of $100 \mu \mathrm{s}$. The method is calibrated using a black-body heat emitter positioned in the test section. The repeatability of the method is within $5 \%$. The numerical procedure proposed by Cook \& Felderman (1966) (see (83) in Schultz \& Jones 1973) for a semi-infinite slab was used to determine heat-flux density $\dot{q}$ at time $t_{n}$ according to

$$
\dot{q}\left(t_{n}\right)=\sqrt{\frac{\rho_{m} c_{m} \lambda_{m}}{\pi}}\left[\sum_{i=1}^{n} \frac{T_{i}-T_{i-1}}{\sqrt{t_{n}-t_{i}}+\sqrt{t_{n}-t_{i-1}}}\right],
$$

where $\rho_{m} c_{m}$ and $\lambda_{m}$ are the density, specific heat capacity and thermal conductivity of the slab material, respectively, and $T_{i}$ is the wall temperature at time $t_{i}$. The same 
(a)

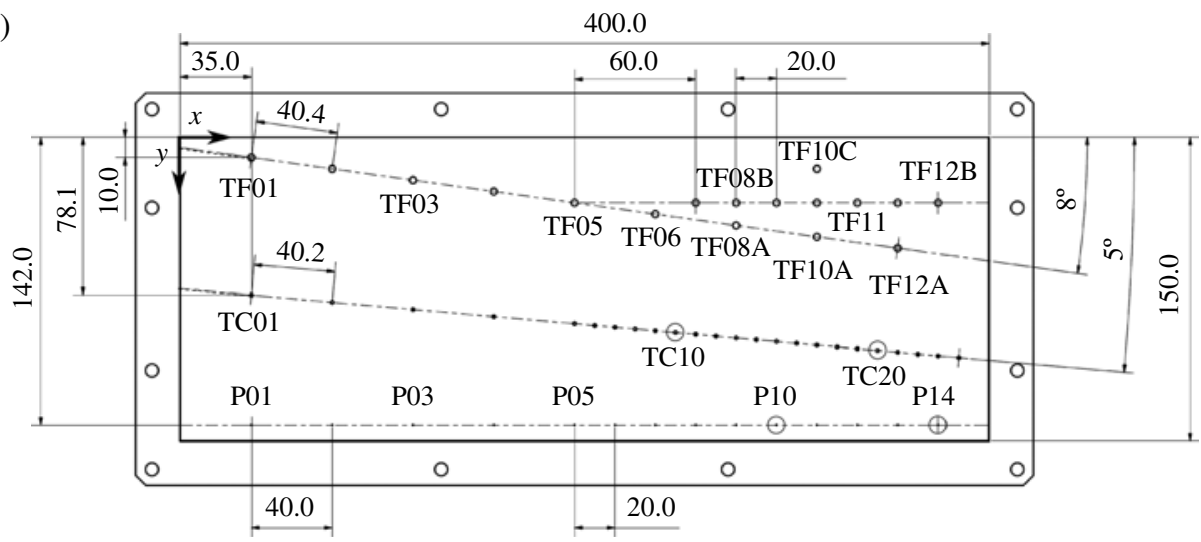

(b)

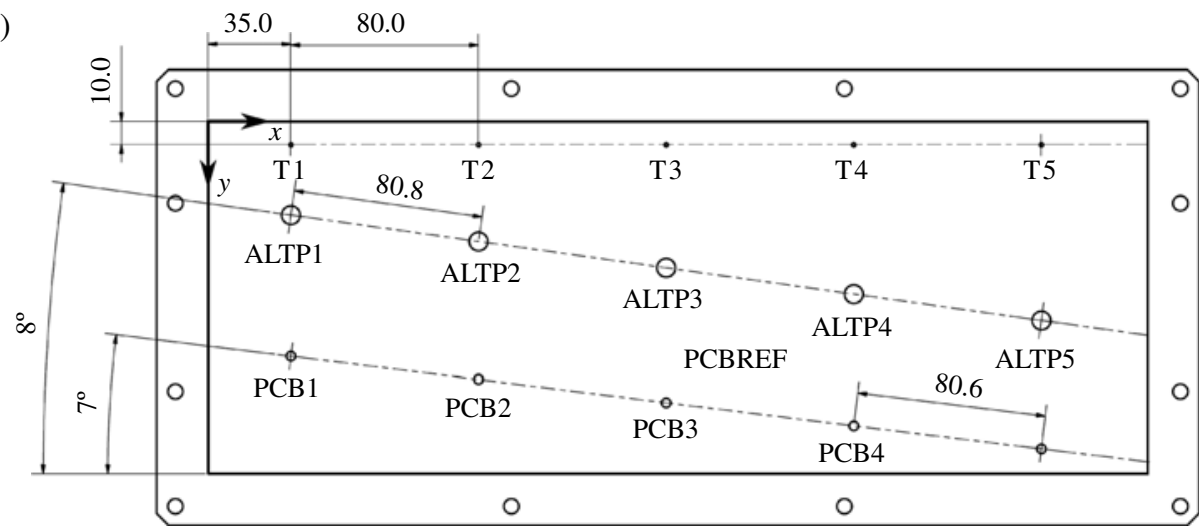

FIGURE 2. Configuration of the plate inserts showing sensor locations $(a)$ insert $\mathrm{T}$ and $(b)$ insert $\mathrm{P}$, showing the locations of thin film (TF), thermocouple (TC, T), Kulite pressure (P), PCB pressure (PCB) and ALTP sensors. Dimensions are in millimetres.

method was used in the $\mathrm{H} 2 \mathrm{~K}$ facility except that the frame rate was $60 \mathrm{~Hz}$ and the exposure time was $15 \mathrm{~ms}$.

Heat flux is also measured using thin film and thermocouple gauges. The thin film sensors (Manufactured by the French-German Research Institute ISL, Saint Louis, France) provide a very high sensitivity of measurement, since surface temperature changes of the order of $0.1 \mathrm{~K}$ can be measured with a response time of microseconds. By contrast the thermocouple sensors (manufactured by MEDTHER Corporation, Huntsville, AL, USA) are more robust and easy to operate at extremely high level of heat flux, but are one order of magnitude less sensitive. Fast response piezoelectric pressure sensors, manufactured by $\mathrm{PCB}$, have response times of the order of microseconds with a natural frequency larger than $1 \mathrm{MHz}$. Kulite pressure transducers are used in this study to measure the mean pressure distribution.

A novel heat flux sensor for transient measurements is the ALTP fast-response sensor (manufactured by FORTECH GmbH, Germany). Its working principle is based on temperature difference measurements in very thin films using the transverse Seebeck effect (Renk et al. 1994; Knauss et al. 2009). The advantages of the ALTP sensor arise from the very thin sensing element (down to 10-100 nm), giving bandwidths up to $1 \mathrm{MHz}$ (Renk et al. 1994). In addition, the sensors do not require 
heating and do not produce thermal disturbances that might lead to interference effects when using arrays of sensors. The conclusion from Knauss et al. was that the sensor is suitable for boundary layer stability studies in short-duration as well as in high-enthalpy facilities.

\subsection{Numerical simulations}

The DNS code has been used over a number of years at the University of Southampton for studies of instability, transition and turbulence in high-speed flows. Here we provide only a short recap of the principal features of the code and explain the run conditions for the present study. Details of the governing equations and algorithm can be found in Touber \& Sandham (2009) and the references cited therein. The numerical method solves the full compressible Navier-Stokes equations using an entropy-splitting approach for the Euler terms and uniformly fourth-order accurate finite differences. Viscosity varies with temperature according to Sutherlands law with a reference temperature set as $65 \mathrm{~K}$ to match the RWG flow condition and the Sutherland constant set to $110.4 \mathrm{~K}$. Shocks are captured using a scheme that combines a compressive limiter with additional sensors to limit the additional dissipation to the immediate vicinity of a shock wave and to turn off the shock capturing within vortical flow regions. In the present work no subgrid model is used, and we use a fine grid so that we can class the simulations as DNS. The hardest part of the flow to resolve is the near-wall region after reattachment, where the highest skin-friction and heat-transfer coefficients are observed. For the worst-case (high disturbance amplitude) simulation the grid in this region has $\Delta x^{+}=\Delta z^{+}=4.6$ and the first off-wall grid point is at $y^{+}=0.6$, where a superscript ${ }^{+}$denoted normalisation with the wall shear stress and fluid properties. The grid suitability for the present simulations was also verified by grid refinement in all directions. The most sensitive feature was found to be the peak skin friction and peak heat flux which varied by less than $5 \%$ and $10 \%$, respectively, for a grid that was coarsened by $50 \%$ in all directions, implying a confidence level of $1 \%$ and $2 \%$, respectively, in the same quantities on the fine grid if the fourth order accuracy of the numerical method is used (in practice, a lower global order of convergence is expected when shock waves are present, but the present code resulted from optimisation of the shock capturing approach to retain the benefits of high order, as described by Yee, Sandham \& Djomehri 1999).

The simulations are carried out for dimensionless variables, using the inflow displacement thickness $\delta_{0}^{*}$ as the reference length scale and inflow free-stream properties as the reference for velocity, density and temperature. Dimensionless quantities are specified with hat variables, so that $\hat{x}=x / \delta_{0}^{*}, \hat{u}=u / U_{\infty}, \hat{\rho}=\rho / \rho_{\infty}$, etc. Pressure is normalised as $\hat{p}=p /\left(\rho_{\infty} U_{\infty}^{2}\right)$ and time as $\hat{t}=t U_{\infty} / \delta_{0}^{*}$. The computational domain is shown in figure 3 . The inflow is downstream of the plate leading edge. At this location a similarity solution of the laminar compressible boundary-layer equations (White 2006; see also appendix A of the current article) is imposed and disturbances (denoted with a prime) are added to the density following

$$
\hat{\rho}^{\prime}=a W(\hat{y}) \sum_{j=1}^{J} \cos \left(\frac{2 \pi j \hat{z}}{\hat{L}_{z}}+\phi_{j}\right) \sum_{k=1}^{K} \sin \left(2 \pi \hat{f_{k}} \hat{t}+\psi_{k}\right),
$$

where $a$ is the amplitude, $\hat{L}_{z}$ is the spanwise domain width and the phases $\phi_{j}$ and $\psi_{k}$ are set to random numbers between 0 and $2 \pi$. Unless otherwise stated, the frequencies are set to $\hat{f}_{k}=0.02 k$, with $J=16$ and $K=20$. It should be noted that the random 


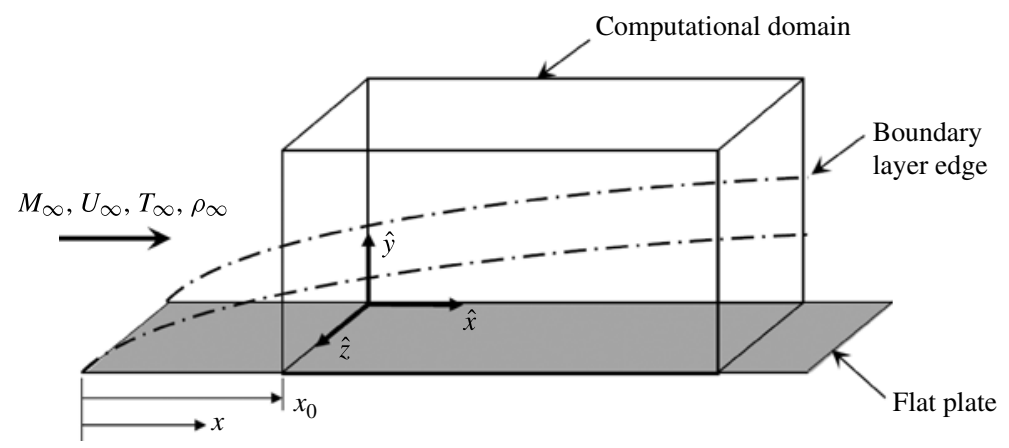

FIgURE 3. Computational domain for numerical simulations. Computational coordinates are related to the start of the computational domain and normalised with $\delta_{0}^{*}$, the displacement thickness at $x=x 0$, where $x$ is measured from the plate leading edge.

phases are fixed for the entire simulation (and indeed are the same for all simulations presented here). The root-mean-square disturbance amplitude is approximately nine times the amplitude parameter $a$. A window function $W(\hat{y})$ is used to remove the initial disturbances from parts of the flow. In the current study the disturbances are windowed near the upper boundary and near the test wall to zero at the wall using $W(\hat{y})=1-\exp \left(-\hat{y}^{3}\right)$. This windowing adds disturbances inside the boundary layer at the inflow. The disturbances are the same as used in De Tullio (2013) where they were classed by type A (acoustic) disturbances and were effective at triggering transition behind roughness elements. For $a=0.001$ (denoted as the high disturbance case in the following) the root-mean-square stagnation pressure was computed to be $3.82 \%$ (a factor of two above the RWG2 measurement) and the root-mean-square streamwise velocity as $0.56 \%$, with these value decreases proportionally for lower values of $a$.

The remaining boundary conditions are set as a solid wall (lower boundary) with no-slip and fixed temperature conditions. The ratio of the wall temperature to free-stream temperature is set to 4.5 to match the RWG conditions. The outflow boundary is treated with a standard characteristic scheme (Thompson 1987). The upper boundary has a fixed reference condition including the shock jump conditions necessary to impose the inviscid shock arising from a $4^{\circ}$ turning angle. Superimposed on this are the integrated outgoing characteristic waves, giving the upper boundary also a low-reflection property. The DNS cases discussed in this paper are listed in table 1. The disturbance amplitude is varied by changing $a$ in (2.2). The high-, medium- and low-amplitude cases listed in table 1 correspond to $a=0.001,0.0005$ and 0.00025 . The Reynolds number is based on the displacement thickness at the inflow to the computational domain and the free-stream properties. The table also includes the inviscid shock impingement point and the Reynolds number based on the distance from the leading edge of the plate to the inviscid shock impingement point (assuming a similarity solution all the way to the leading edge). The streamwise domain length is set to $\hat{L}_{x}=300$, the wall-normal to $\hat{L}_{y}=25$ and the spanwise to $\hat{L}_{z}=45$. One simulation (not shown here) was run with a wider domain that showed Stanton number variations within $1 \%$, so the present domain is believed to be wide enough. The grid contains a total of 200 million grid points for each simulation $(2401 \times 232 \times 360$ in the $\hat{x}, \hat{y}, \hat{z}$ directions, respectively) and the simulations were run on 8192 cores of the HeCTOR supercomputer. The simulations were run in two 


$\begin{array}{lccccc}\text { Case } & \text { Shock } & \text { Amplitude } & \hat{x}_{i m p} & \operatorname{Re}_{x_{i m p}} & \text { Computational grid } \\ \text { NS-H } & \text { No } & \text { High } & - & - & 2401 \times 232 \times 360 \\ \text { NS-M } & \text { No } & \text { Medium } & - & - & 2401 \times 232 \times 360 \\ \text { NS-L } & \text { No } & \text { Low } & - & - & 2401 \times 232 \times 360 \\ \text { S-H } & \text { Yes } & \text { High } & 150 & 1.338 \times 10^{6} & 2401 \times 232 \times 360 \\ \text { S-M } & \text { Yes } & \text { Medium } & 150 & 1.338 \times 10^{6} & 2401 \times 232 \times 360 \\ \text { S-L } & \text { Yes } & \text { Low } & 150 & 1.338 \times 10^{6} & 2401 \times 232 \times 360 \\ \text { S-HU } & \text { Yes } & \text { High } & 115 & 1.099 \times 10^{6} & 2401 \times 232 \times 360 \\ \text { S-HD } & \text { Yes } & \text { High } & 185 & 1.577 \times 10^{6} & 2401 \times 232 \times 360\end{array}$

TABLE 1. Numerical simulation cases, all at $M=6, R e=6830, T_{w} / T_{\infty}=4.7, \mathrm{~S}=$ shock, $\mathrm{NS}=$ no shock, $\mathrm{H}=$ high, $\mathrm{M}=$ medium, $\mathrm{L}=$ low, $\mathrm{U}=$ upstream, $\mathrm{D}=$ downstream.

stages, first to clear transient features and then to accumulate flowfields and statistical data over one cycle of the lowest frequency of forcing.

The main dimensionless properties of interest are the skin friction coefficient $c_{f}$ and Stanton numbers $S t$ defined by

$$
c_{f}=\frac{\mu_{w}}{\frac{1}{2} \rho_{\infty} U_{\infty}^{2}}\left(\frac{\mathrm{d} u}{\mathrm{~d} y}\right)_{w}
$$

and

$$
S t=\frac{\kappa_{w}}{\rho_{\infty} U_{\infty} c_{p}\left(T_{r}-T_{w}\right)}\left(\frac{\mathrm{d} T}{\mathrm{~d} y}\right)_{w},
$$

respectively, where a subscript $w$ denotes conditions on the wall $(\hat{y}=0)$ and subscript $\infty$ denotes free-stream conditions at a reference location upstream of any shock impingement. The recovery temperature is given by

$$
\frac{T_{r}}{T_{\infty}}=1+r \frac{\gamma-1}{2} M_{\infty}^{2},
$$

with $r=\operatorname{Pr}^{1 / 2}$ for laminar flow and $r=\operatorname{Pr}^{1 / 3}$ usually assumed for turbulent flow. Note that for the transitional cases reported here we define $S t$ using the laminar recovery factor. Reference laminar and turbulent solutions are provided in appendix A.

\section{Flow visualisation and mean Stanton number distributions}

In this section we provide an overview of the interaction and demonstrate the level of agreement between experiment and DNS, focusing on the RWG experimental results, but including some $\mathrm{H} 2 \mathrm{~K}$ data. Figure 4 shows a pseudo-schlieren $(|\nabla \rho|)$ plot from the simulation and an experimental shadowgraph (from phase 1 of the RWG experiments), where we have zoomed in on the interaction region. The images are aligned using the trailing edge of the shock generator, located at $x=0.245 \mathrm{~m}$, and the experimental shock impingement location at $x=0.324 \mathrm{~m}$. The experimentally measured impingement location is approximately $0.01 \mathrm{~m}$ upstream of that calculated using the inviscid relations due to viscous effects on the shock generator. It can be seen that there is good agreement in the structure of the interaction, including the location of the edge of the boundary layer, the locations of the reflected shock waves and the point of boundary-layer transition as indicated by the blurring of 
(a)
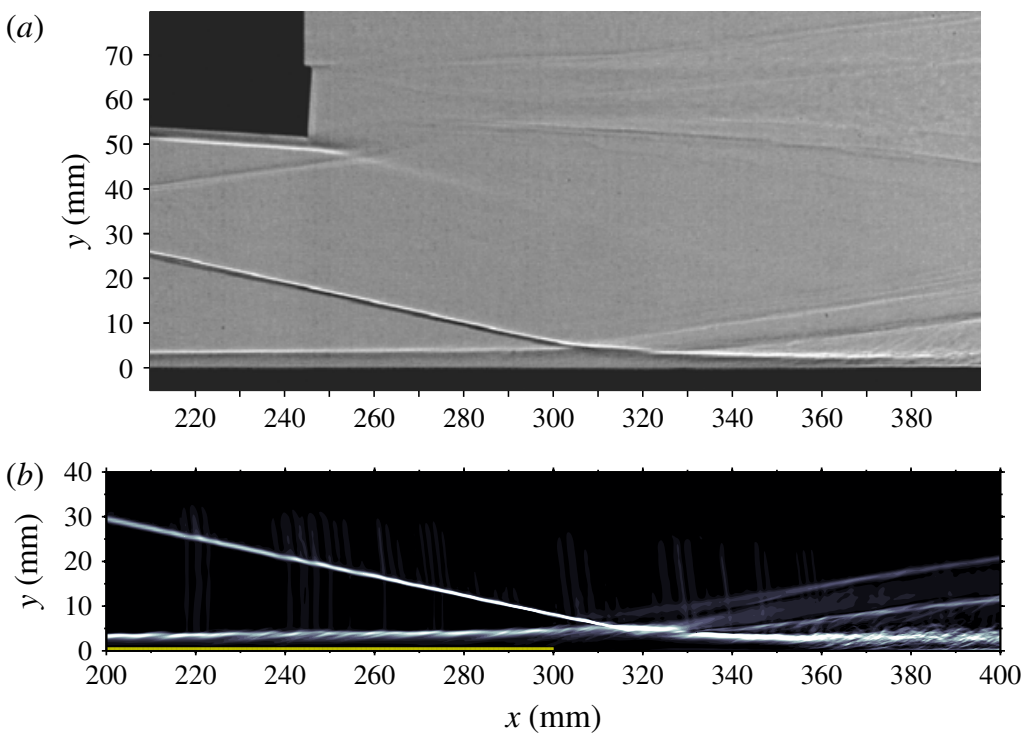

FIgURE 4. (Colour online) (a) Shadowgraph from the RWG experiments at a unit Reynolds number of $R e_{1}=3.99 \times 10^{6} \mathrm{~m}^{-1}$, corresponding to $R e_{i m p}=1.345 \times 10^{6}$ compared with $(b)$ pseudo-schlieren $(|\nabla \rho|)$ contours from one span-averaged realisation of the simulation with high-amplitude forcing (case $\mathrm{S}-\mathrm{H}$ ).

the contours compared with laminar flow regions. Upstream of the interaction the simulated pseudo-schlieren also shows the presence of the large-amplitude inflow disturbances which propagate downstream as acoustic waves with wavefronts that are nearly normal to the flat plate.

A more quantitative comparison is made for Stanton number in figure 5. The plot includes QIRT data from the RWG and H2K experiments compared with DNS for the high- and medium-amplitude cases for experimental flow conditions selected such that the impingement Reynolds number was close to the simulated case of $R e_{i m p}=1.338 \times 10^{6}$. For cases with shocks, the unit Reynolds numbers were 4.0 and $6.1 \times 10^{6} \mathrm{~m}^{-1}$ for the RWG experiments and $6.27 \times 10^{6} \mathrm{~m}^{-1}$ for $\mathrm{H} 2 \mathrm{~K}$ (higher since the shock impingement was at $228 \mathrm{~mm}$ compared with the $337 \mathrm{~mm}$ in the RWG1 experiment). Corresponding values for the no shock case were $3.55 \times 10^{6} \mathrm{~m}^{-1}$ for the RWG1 experiment, $4.0 \times 10^{6} \mathrm{~m}^{-1}$ and $6.1 \times 10^{6} \mathrm{~m}^{-1}$ for RWG2 and $6.18 \times 10^{6} \mathrm{~m}^{-1}$ for H2K. Figure 5(a) shows the comparison without shock impingement. It can be seen that the DNS high-amplitude case is close to the RWG experiments, despite the higher levels of stagnation pressure fluctuations in the DNS compared with the RWG measurements, while the medium-amplitude case is close to the $\mathrm{H} 2 \mathrm{~K}$ experiment. This is not the entire picture, since it has already been remarked that the disturbance levels reduce in RWG with increasing $R e_{1}$ so that the transition point in RWG is expected to move to higher $R e_{x}$ with an increase in $R e_{1}$. The two phases of the RWG experiment show similar behaviour, with more data obtained downstream in RWG2 that shows a significant overshoot relative to the turbulent correlation. The fact that similar behaviour has been seen in RWG and DNS despite the differences in free-stream disturbances suggests that the forcing method used in DNS does not quantitatively reproduce the free-stream disturbance amplitudes in the experiment. More work is needed in the future to develop methods of imposing fluctuations 

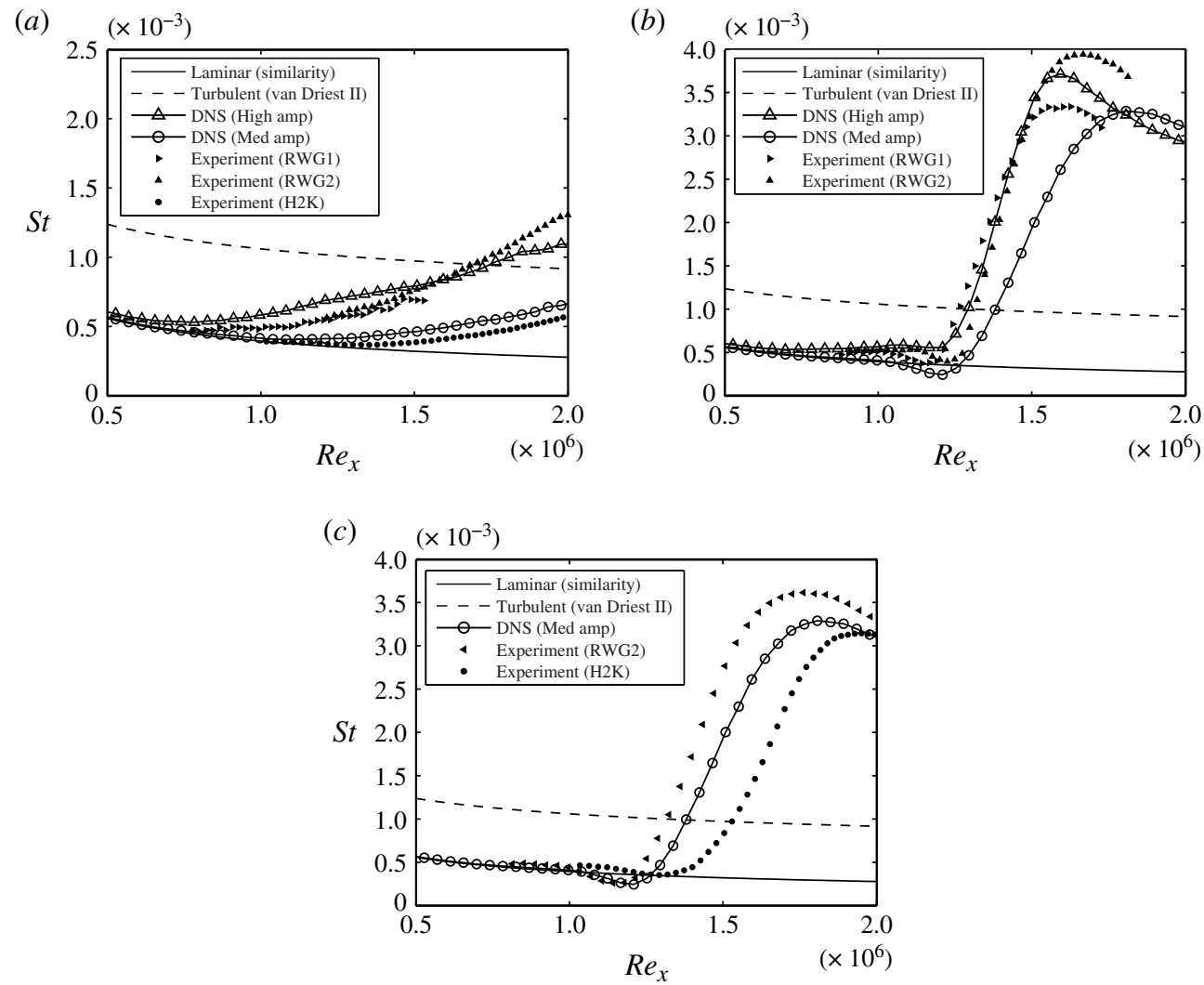

FIgURE 5. Comparison of $S t$ distributions at nominally the same Reynolds number $R e_{i m p}$ based on distance to shock impingement and flow conditions: $(a)$ cases without shock impingement; $(b)$ cases with shock impingement at $R e_{1}=4 \times 10^{6} \mathrm{~m}^{-1}$; and (c) cases with shock impingement at $R e_{1}=6 \times 10^{6} \mathrm{~m}^{-1}$. Large open symbols correspond to DNS cases NS-H and NS-M in $(a)$ and S-H and S-M in (b). Small symbols correspond to the RWG and $\mathrm{H} 2 \mathrm{~K}$ experiments. For improved readability of the figure, only every sixth data point is shown from RWG, every second data point from $\mathrm{H} 2 \mathrm{~K}$ and every 50th grid point from DNS. The dashed line is the Van Driest II turbulent correlation (appendix A) and the solid line is the laminar similarity solution.

that are representative of experiment and also in experiments to provide a more complete measurement of different aspects of the fluctuations (including spectra and lengthscales as well as amplitude).

Figure 5(b) compares cases with shock impingement for the nominal $R e_{1}=$ $4 \times 10^{6} \mathrm{~m}^{-1}$. For these cases there are only minor differences in $R e_{i m p}$, which is $1.338 \times 10^{6}$ for the DNS, compared with $1.345 \times 10^{6}$ for the RWG1 experiments and $1.336 \times 10^{6}$ for the RWG2 experiment. We have already seen that in RWG1 the shock impingement measured from the shadowgraph is slightly (3\%) upstream of the inviscid calculation. Bearing in mind this small correction it can be seen from figure $5(b)$ that the RWG experiments are in excellent agreement with the high-amplitude DNS case. The onset of transition is similar and the rate of increase in $S t$ is similar. The peak $S t$ from the simulation falls between the experimental 

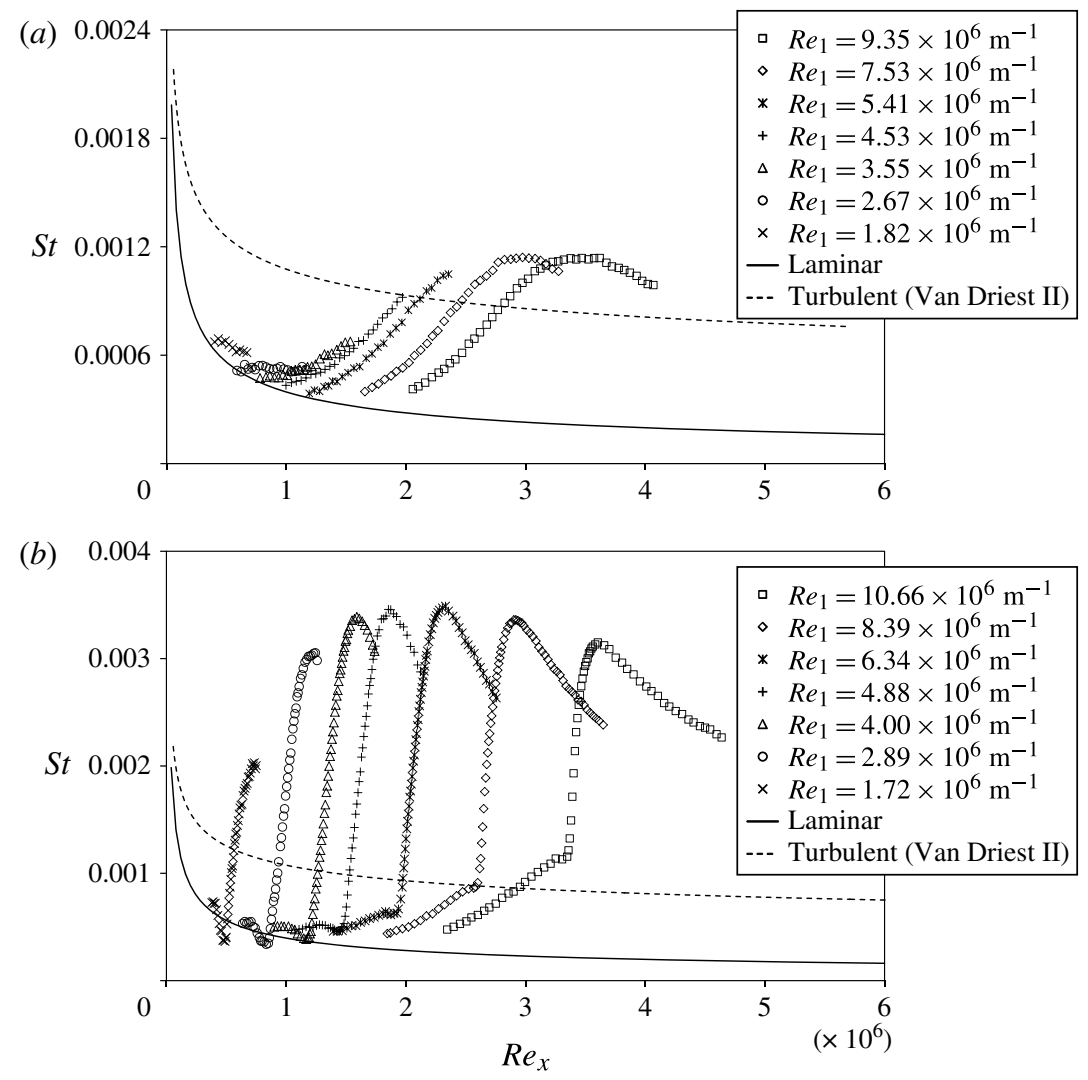

FIGURE 6. RWG (phase 1) QIRT results for a scan over a wide range of $\operatorname{Re}_{1}(a)$ without and $(b)$ with shock impingement $\left(S t\right.$ versus $\left.R e_{x}\right)$.

data (0.003 75 compared with 0.0034 from RWG1 and 0.0040 from RWG2) but the undershoot in $S t$ that precedes the rapid rise is smaller in the DNS.

Figure $5(c)$ compares the RWG2 and $\mathrm{H} 2 \mathrm{~K}$ cases for the same nominal $R e_{1}$ $\left(6.1 \times 10^{6} \mathrm{~m}^{-1}\right.$ in RWG and $6.18 \times 10^{6} \mathrm{~m}^{-1}$ in $\left.\mathrm{H} 2 \mathrm{~K}\right)$ and approximately the same shock impingement location ( $214 \mathrm{~mm}$ in RWG2 and $228 \mathrm{~mm}$ in H2K). The shock impingements are not exactly matched in this case, but even if one makes a slight adjustment, for example by aligning on the $S t$ minima, there is still a delay in the transition in $\mathrm{H} 2 \mathrm{~K}$ compared with RWG suggesting a lower free-stream disturbance level. The DNS medium-amplitude case bisects the two experiments, in contrast to the agreement with the high-amplitude case at the lower $R e_{1}$, again suggesting a reduced disturbance level at the higher $R e_{1}$. Taking into account all of the results shown in figures 4 and 5 the comparison with experimental data is sufficiently good to allow the simulation approach to be used to study other aspects of the transitional interactions and extract additional data not available from the experiments.

The RWG (phase 1) experiments included a sweep over a wide range of unit Reynolds numbers. The resulting $S t$ distributions for cases without a shock impingement are shown in figure 6(a) compared with reference laminar and turbulent relations. The abscissa is shown as the Reynolds number based on distance from the plate leading edge, while the numbers shown in the legend indicate the unit Reynolds number for the individual wind tunnel runs. At the lowest unit Reynolds numbers the 
flow is entirely laminar while at the highest values the flow is transitional from the start of the measurements. In addition, it can be seen how the transition Reynolds number increases with increasing unit Reynolds number. The present observations are consistent with previous measurements, for example Pate \& Schueler (1969), which noted an increase in transition Reynolds number with tunnel size and explained this by the decrease in acoustic fluctuations. The need to take into account the disturbance environment in hypersonic facilities, including in particular the disturbance spectrum, was recognised by Reshotko (1969). In this connection, Arnal \& Delery (2004) note that as the unit Reynolds number increases, the range of unstable waves shifts to higher frequencies, for which the free-stream pressure disturbances are smaller.

Figure $6(b)$ shows the equivalent results with shock impingement. In these cases there is a significant overshoot in Stanton number, well above the level found in turbulent boundary layers. The correlation of Neumann (1972) would lead to a factor of 2.5 increase in heat flux, above the turbulent flat plate case, whereas here we have factors of 3.5 relative to the Van Driest II correlation for a turbulent boundary layer. It can also be seen from the figure that the highest Stanton number is obtained not for the most turbulent interaction at the highest unit Reynolds number, but for the interaction at $R e_{1}=6.34 \times 10^{6} \mathrm{~m}^{-1}$. We shall return to this result after we have reviewed results from DNS. From a comparison of figure $6(a, b)$ it is possible to identify four different zones of the interaction (as may also be inferred from previous studies such as Benay et al. (2006)): (i) the undisturbed flow upstream of the SWBLI where the Stanton number distribution is unaffected by the shock impingement; (ii) a zone with significantly reduced heat transfer, dipping below the laminar boundary-layer solution; (iii) a rapid rise in heat transfer up to a maximum downstream of the shock impingement point; and (iv) a slow recovery of heat-transfer rate towards that of an equilibrium turbulent boundary layer.

A separate study was made in RWG (in phase 2 of the work) of the effect of shock impingement location. The results are shown in figure $7(a, b)$ for unit Reynolds numbers $R e=4.0 \times 10^{6}$ and $6.1 \times 10^{6}$, respectively. As with the sweep over unit Reynolds number, both cases show a peak in the maximum value of $S t$ obtained for cases starting from a transitional value of $S t$ between the laminar and equilibrium turbulent levels. The higher levels of peak $S t$ obtained at the lower unit Reynolds number are believed to be a further consequence of the changing disturbance environment. It was seen in the DNS results shown on figure $5(b)$ that higher disturbance levels led to a higher, but narrower, peak in St.

Using DNS the effect of shock impingement Reynolds number can also be studied, but it is more convenient in this case to fix the inflow Reynolds number and computational domain and vary the shock impingement position as in the RWG2 tests. This allows the impingement to occur at different values of $R e_{x}$ and of the local intermittency. Cases S-HU and S-HD detailed in table 1 are for a shock shifted upstream and downstream, respectively. Cases S-HU, S-H and S-HD form a set of simulations in which the intermittency (anticipating the results of the next section) at the nominal shock impingement location is $25 \%, 43 \%$ and $61 \%$, respectively.

The laminar 2D solution, expressed in terms of the wall pressure, skin friction and Stanton number is shown in figure 8, showing that the basic separation bubble structure is not strongly dependent on $R e_{i m p}$. From the data shown in the figure it can be seen that the bubble is longer when the shock is in its downstream location and shorter when upstream. The minimum value of the skin friction coefficient is similar in all cases.

The wall pressure distributions for the 3D cases with transition to turbulence are shown in figure $9(a)$. There is no pressure plateau in these cases with high disturbance 

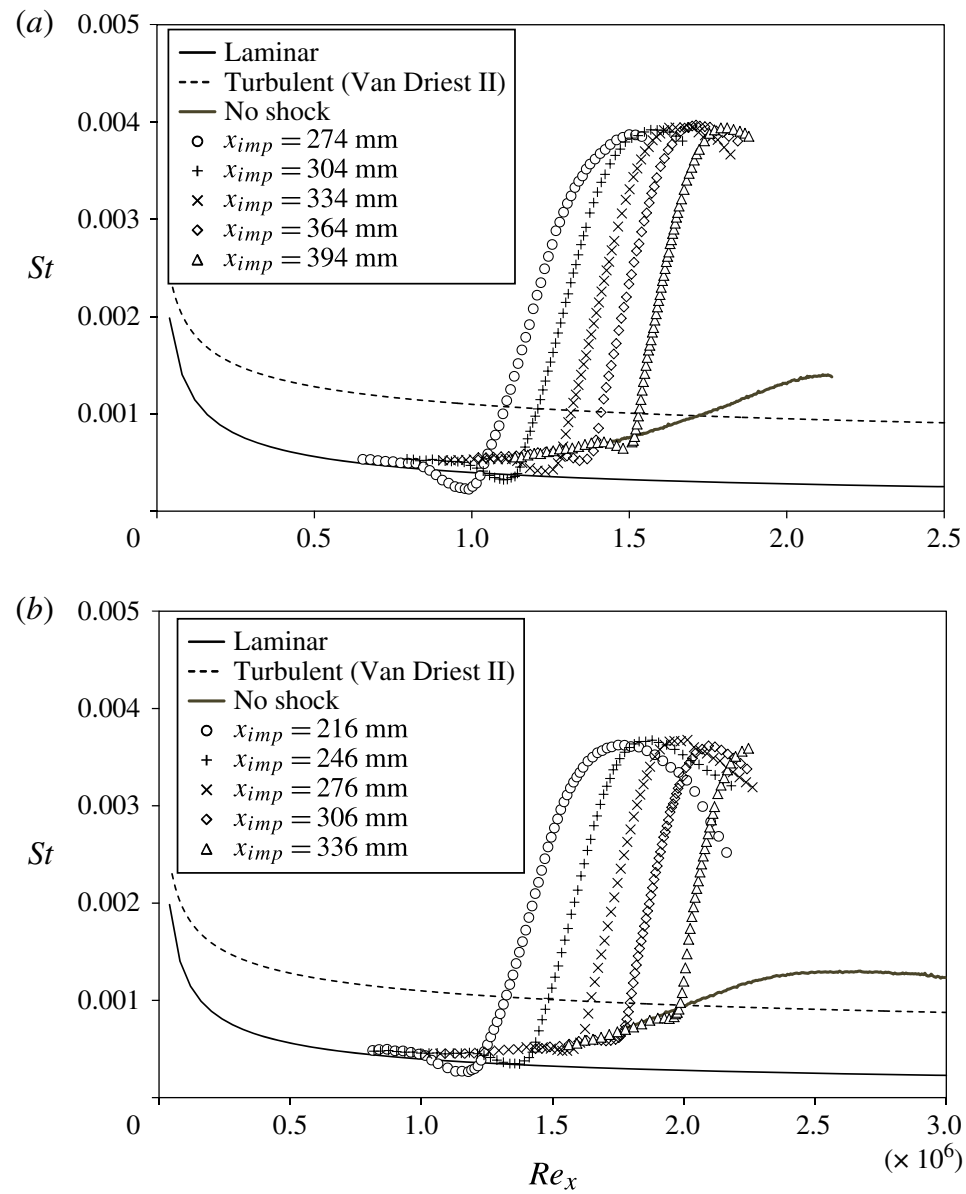

FIGURE 7. RWG (phase 2) QIRT results for a scan over shock impingement location at (a) $R e_{1}=4.0 \times 10^{6} \mathrm{~m}^{-1}$ and (b) $R e_{1}=6.1 \times 10^{6} \mathrm{~m}^{-1}$ (St versus $R e_{x}$ ).

amplitude and the small kink in the curve that is the precursor to a plateau is only noticeable when the shock is in its upstream and centred locations. As shown in figure $9(b)$, the upstream shock location is the one that has the strongest effect on the skin friction and the only case where the time- and span-averaged skin friction actually becomes negative. The Stanton number distribution in figure $9(c)$ shows similar behaviour and includes a comparison with RWG2 data for the same upstream, downstream and centred shock impingement locations. The peak Stanton number is slightly higher for the centred shock location, in agreement with the experiments. The experiments tend to show a lower dip in $S t$ at the start of the interaction and a delayed broader peak, but the maximum rate of increase of $S t$ is similar and overall the agreement with the experimental data is excellent.

Using DNS it is possible to make a quantitative assessment of Reynolds analogy between $c_{f}$ and $S t$ that would commonly be assumed as a constant in empirical correlations and in turbulence modelling. In figure 10 the quantity $c_{f} /(2 S t)$ is plotted as a function of the downstream distance. The standard Reynolds analogy would set this ratio to $\operatorname{Pr}^{2 / 3}(\approx 0.8)$ as was done to get the turbulent $S t$ correlation in appendix A. From figure 10 we can see that the DNS data show that the analogy 

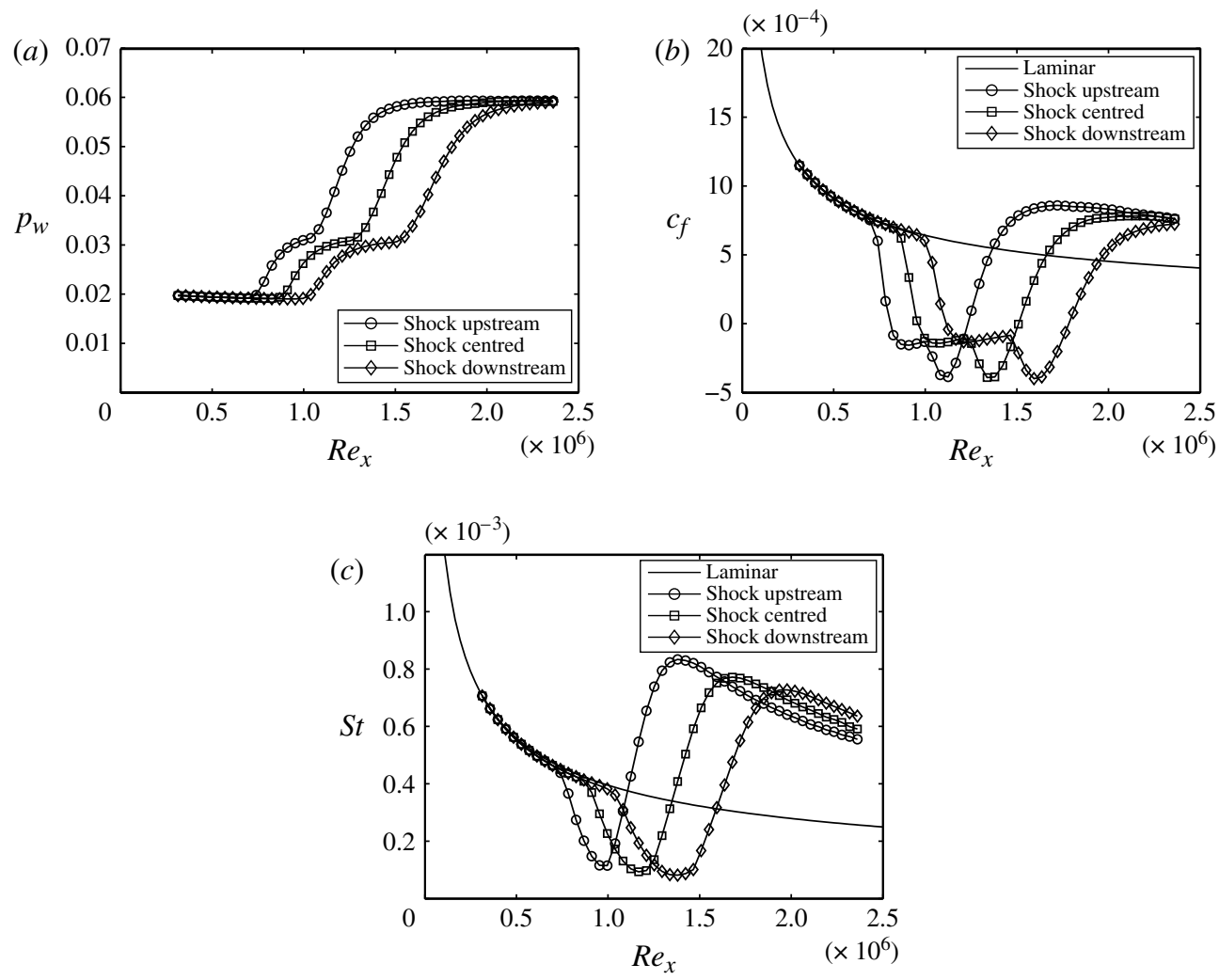

FIGURE 8. Laminar interaction based on 2D simulations with three different shock impingement locations (cases S-HU, S-H and S-HD): (a) wall pressure $p_{w}$, (b) skin friction coefficient $c_{f}$ and $(c)$ Stanton number St. The solid line in $(b)$ and $(c)$ shows the laminar similarity solution.

is, as expected, very poor through the interaction zone, while downstream of the interaction a Reynolds analogy factor of around $2 / 3$ is seen in the DNS results downstream of reattachment, implying a subsequent long relaxation back to the typical factors of $0.83-0.85$ seen in DNS of fully developed turbulent flow at high speed (Duan \& Martin 2011).

To summarise the results of this section, figure 11 shows the combined results from RWG2, DNS and H2K plotted in the form of $S t_{\max } / S t_{\text {lam }}$ against $\left(S t_{t r}-S t_{\text {lam }}\right) /\left(S t_{\text {tur }}-\right.$ $\left.S t_{\text {lam }}\right)$. In this plot, $S t_{\max }$ is the maximum $S t, S t_{\text {lam }}$ is $S t$ for a laminar boundary layer at the inviscid shock impingement point (with $S t_{t u r}$ for a similarly defined turbulent case, using the Van Driest II correlation) and $S t_{t r}$ is the measured $S t$ of the transitional boundary layer at the interaction point but without SWBLI. For purely laminar flow the values of $S t_{\max } / S t_{\text {lam }}$ is approximately 2.1 taken from the DNS results shown in figure $8(\mathrm{c})$. This value is very close to the ratio across the impinging shock of $\rho_{2} U_{2} / \rho_{1} U_{1}=2.09$, which would be the change in Stanton number of a laminar boundary layer but with the denominator in (2.4) taken from the post-shock conditions. The abscissa in figure 11 may be thought of as a measurement of the state of the boundary layer at the nominal impingement location, equal to zero for fully laminar flow and increasing to values of one for a fully turbulent interaction, which can be related to the boundary layer intermittency. However, it should be noted that the 
(a)

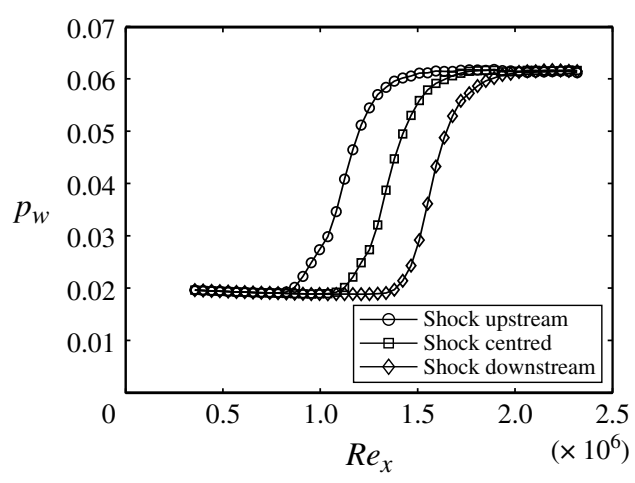

(b)

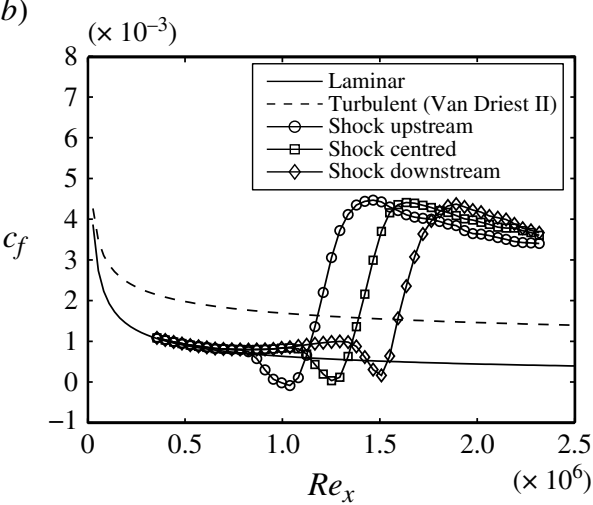

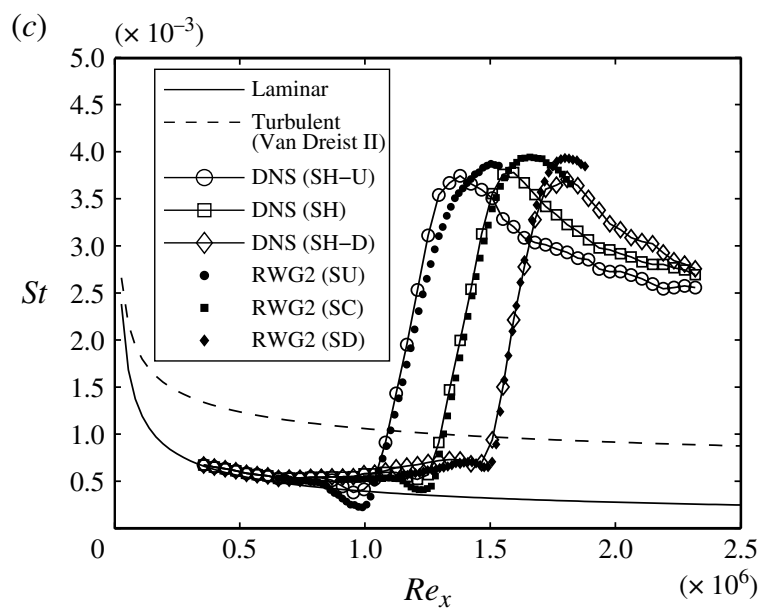

FIGURE 9. Transitional interactions based on 3D simulations with three different shock impingement locations (cases S-HU, S-H and S-HD): (a) wall pressure $p_{w},(b)$ skin friction coefficient $c_{f}$ and $(c)$ Stanton number $S t$, including a comparison with experimental cases with shock impingement $(\mathrm{SU}=$ shock upstream, $\mathrm{SC}=$ shock centred and $\mathrm{SD}=$ shock downstream). To improve readability, only every 6th RWG2 data point and every 50th DNS grid point are shown. The solid line in $(b)$ and $(c)$ shows the laminar similarity solution and the dashed line shows the Van Driest II turbulent correlation (appendix A).

definition allows for values greater than one due to the overshoot in $S t_{t r}$ relative to $S t_{\text {tur }}$. The H2K data shows a falling trend as the abscissa increases towards 1, but there is insufficient data in this region of the plot to form a definite conclusion. The main feature of figure 11 is the rapid rise in $S t_{\max }$ above the laminar value for only small increases in the intermittency of the boundary layer, followed by a more gradual increase.

Finally in this section we comment on the effect of leading edge bluntness. In RWG and $\mathrm{H} 2 \mathrm{~K}$ the leading edge was sharp, but in HEG it was seen that a sharp leading edge gave too early a transition, making it difficult to make measurements. Figure 12 shows the heat flux measured by thermocouples for a case with no shock wave. It can be seen that there is a large shift in the transition point between the cases with a leading edge radius of 0.1 and $0.16 \mathrm{~mm}$, followed by a slow streamwise shift of the 


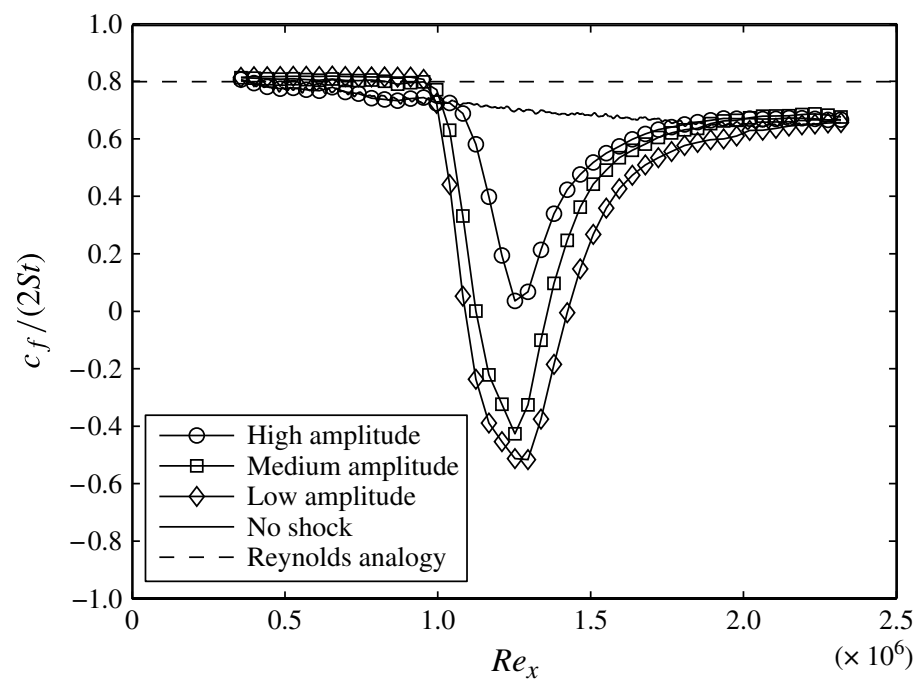

FigurE 10. Test of Reynolds analogy using DNS data for cases S-H, S-M, S-L and NS-H. The dashed line shows the $\operatorname{Pr}^{2 / 3}$ value that is correct for laminar flow.

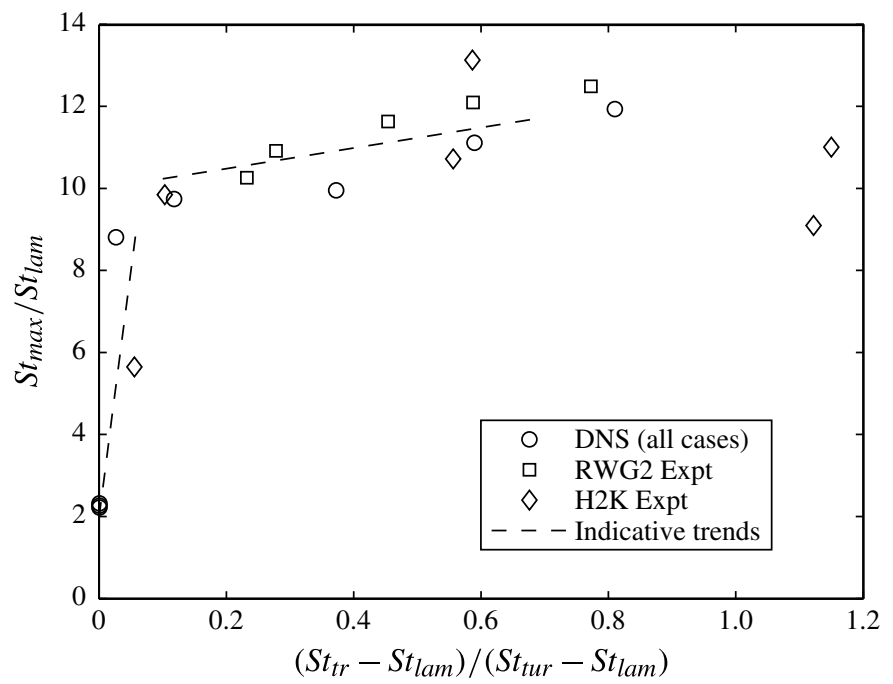

FIGURE 11. Plot of $S t_{\max } / S t_{\text {lam }}$ (measuring the size of the overshoot in heat transfer) against $\left(S t_{t r}-S t_{\text {lam }}\right) /\left(S t_{\text {tur }}-S t_{\text {lam }}\right)$ (measuring the transitional stage of the boundary layer at the interaction location) combining data from RWG, H2K and DNS, with dashed lines showing the indicative trends.

transition point with increasing leading edge radius of curvature. Since the sharpest leading edge would not allow transitional effect to be studied, the leading edge radius was set to $0.16 \mathrm{~mm}$ for the HEG experiments.

\section{Boundary-layer intermittency}

Various methods were tested to extract the boundary-layer intermittency, including direct detection methods and p.d.f.-based method. The preferred method was found to depend on the facility and measurement technique, so we begin this section by 


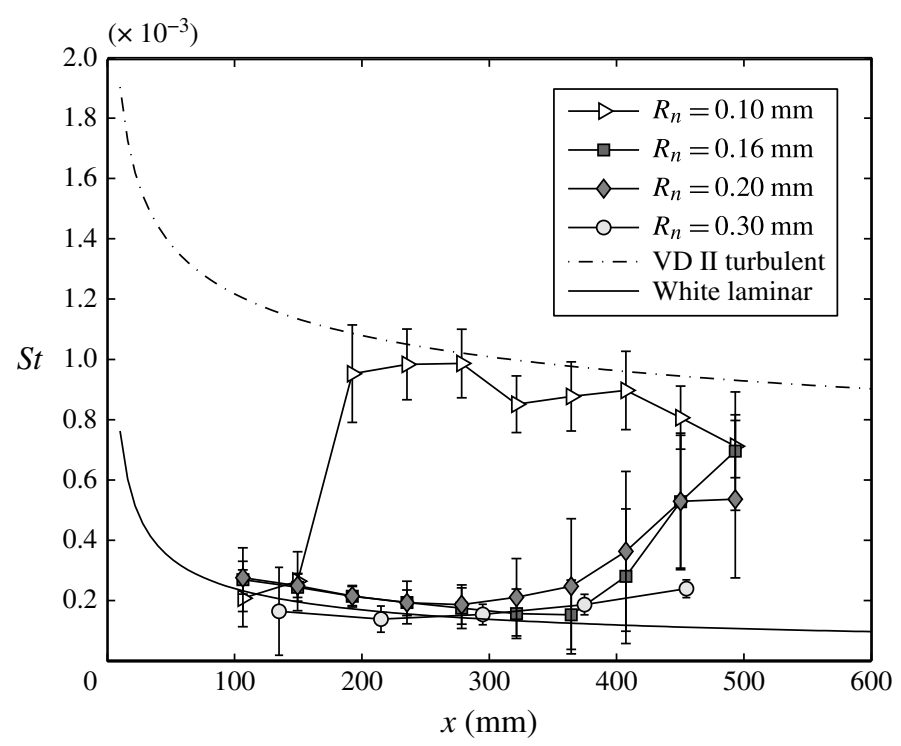

FIGURE 12. HEG heat flux distributions, showing the influence of leading edge radius $R_{n}$.

reporting some of the experiences of attempting to measure intermittency in the different hypersonic flow facilities. In the RWG tests, which were used to scope out the transition behaviour ahead of tests in $\mathrm{H} 2 \mathrm{~K}$ and $\mathrm{HEG}$, useful results in terms of signal-to-noise ratio were obtained only with the ALTP sensors. The other sensors (TC, TF and PCB) are not inherently noisy, but the noise levels could not be satisfactorily reduced in the limited testing time available. Using the single ALTP sensor at different locations it was nevertheless possible to observe intermittent behaviour in the time series. Direct detection methods based on a short time-averaged detector function (see, e.g., Hedley \& Keffer 1974) were found to be robust once the detector function had been optimised. The preferred detector function was based on $\dot{q} \mathrm{~d} \dot{q} / \mathrm{d} t$, where $\dot{q}$ is the measured heat flux density $\left(\mathrm{W} \mathrm{m}^{-2}\right)$. This is similar to the approach proposed by Canepa, Ubaldi \& Zunini (2002) except that they used $\left(e^{2}-e_{0}^{2}\right) / e_{0}^{2}$ derived from a hot film signal $e$ with mean $e_{0}$, which is related, but not equal, to the wall shear stress. In all cases a threshold $S$ must be manually set to distinguish turbulent flow. Window times $t_{\text {win }}=100 \mu \mathrm{s}$ were used with a threshold set typically to $400 \mathrm{~W}^{2} \mathrm{~m}^{-4} \mathrm{~s}^{-1}$. By contrast the p.d.f. method of Schneider (1995) was difficult to apply due to the laminar and turbulent peaks merging together due to the noisy characteristics of the signals and, as seen in the DNS results that will be presented later, high-amplitude low-frequency signal components.

In H2K intermittency was estimated using PCB and ALTP sensors. Signals obtained from thin film gauges were again found to be too noisy to be useful. Measurements with PCB and ALTP sensors also have a high degree of uncertainty, due to the difficulty of calibration and the unknown mechanical oscillation properties of the transducer, but it is possible to detect periods of high fluctuations compared with less noisy times, allowing a measure of intermittency to be extracted. More results from the $\mathrm{H} 2 \mathrm{~K}$ unsteady signals, including a wavelet analysis, are presented in Willems \& Guelhan (2014). Here we show results obtained from a modified p.d.f. method. The signals were considered in $20 \mu \mathrm{s}$ windows in which the inter-quartile range was extracted. A p.d.f. of this quantity was then plotted and a threshold $\left(S_{p}\right.$ for 
(a)

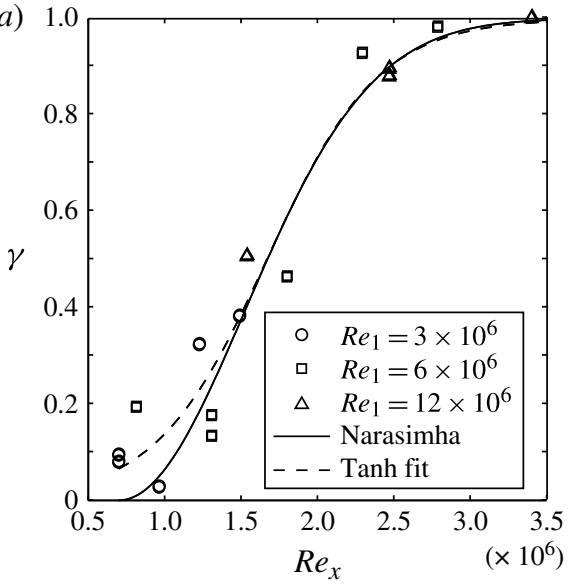

(b)

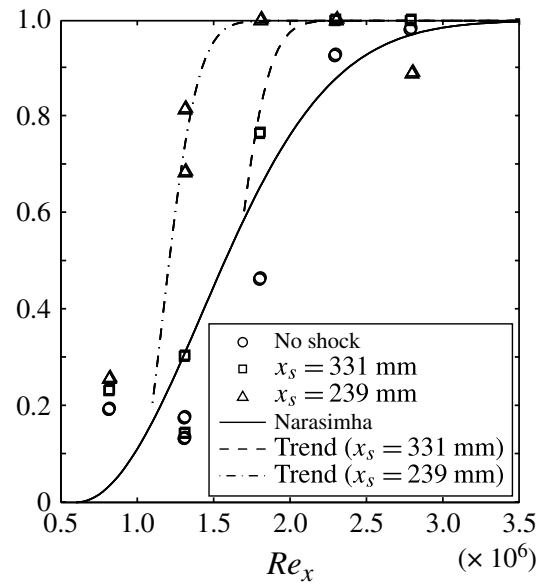

FIGURE 13. Educed intermittency from H2K for cases (a) without shock wave for three different unit Reynolds numbers $R e_{1}$ and $(b)$ with shock wave impingement at two different streamwise locations. The curves are fits to the Narasimha or hyperbolic distributions (solid and dashed lines, respectively), or indicative trends (chain dotted line in $(b))$.

PCB sensors and $S_{q}$ for ALTP heat flux sensors) was used to separate laminar from turbulent regions. Figure 13 shows the resulting intermittency distributions for cases (a) without a shock and $(b)$ with shock impingement at two locations. Thresholds were set as $S_{p}=10 \mathrm{~Pa}(\mathrm{PCB})$ and $S_{q}=1.2 \mathrm{~kW} \mathrm{~m}^{-2}$ ALTP. The solid line is a fit to the data with no shock based on the work of Narasimha (1985), who proposed a formula for intermittency based on the hypothesis of concentrated breakdown, as

$$
\gamma=1-\exp \left[-0.412\left(\frac{H\left(x-x_{T}\right)}{\lambda}\right)^{2}\right]
$$

where $x_{T}$ is the transition onset location, $\lambda$ measures the length of the transition zone and $H$ is the Heaviside function. A first point to make is that there is too much variation within the data to distinguish any clear effect of unit Reynolds number; all the data are well scattered around a Narasimha distribution. At low intermittency levels there is a tendency for the Narasimha formula to underpredict the intermittency and a hyperbolic tangent shape, shown with the dashed line in figure 13(a), is at least as good a fit to the data. A similar observation was made by Steelant \& Dick (2001), where the intermittency-related turbulent spot growth modelling was based upon a exponential-tangent shape near the onset of transition. This was motivated by the natural process of a distributed breakdown near the onset of the transition rather than the assumed concentrated breakdown in the intermittency model (4.1). Results for two cases with shock impingement are shown in figure 13(b) for shock impingement locations $x_{s}=239$ and $331 \mathrm{~mm}$ at $R e_{1}=6 \times 10^{6} \mathrm{~m}^{-1}$, with chain dotted lines showing the trends. It can be seen that following shock impingement the intermittency levels rise rapidly up to the fully turbulent condition $\gamma=1$.

In HEG thermocouples were used to obtain the average Stanton number distributions and thin film gauges were used to extract intermittency (since the heat transfer levels are much higher compared with $\mathrm{RWG}$, the signal-to-noise ratio is improved). A 


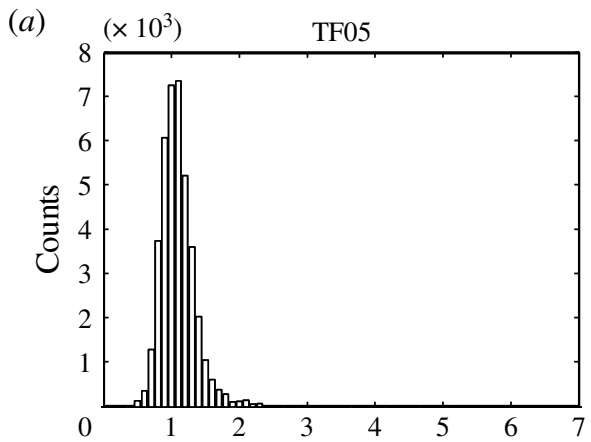

(b)
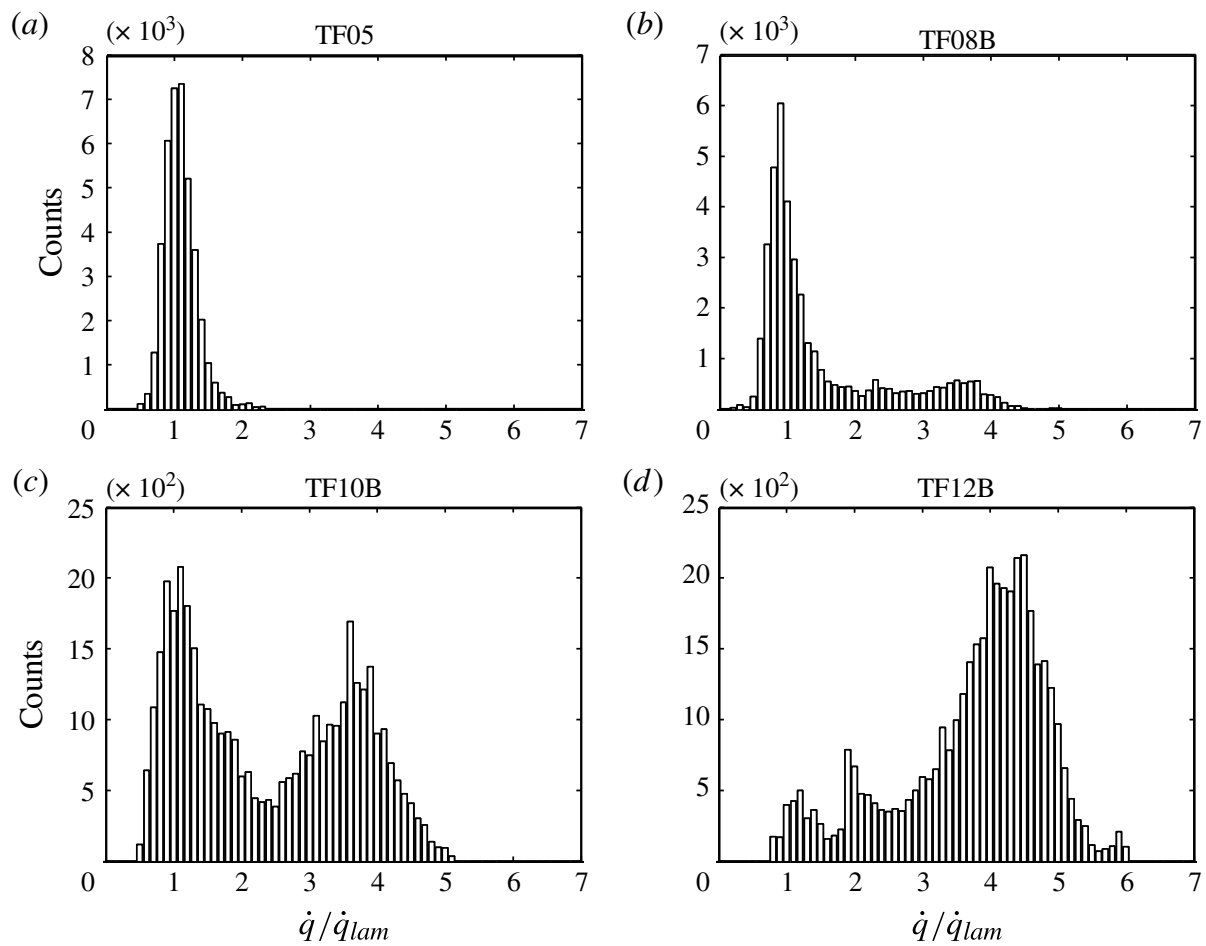

FIGURE 14. A sample of p.d.f.s from HEG, following sensors along a streamwise line and illustrating the change from laminar $(a)$ to turbulent flow $(d)$ via intermediate p.d.f.s (b) and (c) that have two peaks.

smoothing filter (Savitzky \& Golay 1964) was applied to reduce noise, adjusted so that frequencies below $100 \mathrm{kHz}$ are unaffected, while those above $150 \mathrm{kHz}$ are strongly damped. Examination of time traces showed structures propagating downstream in the boundary layer at speeds of $77 \%$ of the free-stream velocity. Such structures were repeatable in other tests and were observed starting from sensor TF07 (figure $2 a$ ). Probability density functions were obtained from the thin-film sensors. A selection is shown in figure 14, showing TF05, TF08B, TF10B and TF12B, which can be seen from figure 2 to be arranged along a streamwise line. The figure shows a clear progression from laminar to nearly fully turbulent flow. In contrast to the other cases, the HEG results at intermediate stations (for example, sensor TF10B) clearly show separate peaks around the laminar and turbulent mean values, indicating the presence of distinct turbulent spots in the flow. Such signals are similar to those observed by Schneider (1995) when the p.d.f. method was proposed and for this case the p.d.f. method is clearly preferred over direct detection methods with greater sensitivity to the choice of thresholds. Nevertheless the p.d.f. method still requires one parameter to be set and after consideration of all of the data, the level of $\dot{q} / \dot{q}_{\text {lam }}=3$ was chosen, where $\dot{q}_{l a m}$ is the heat transfer rate of the undisturbed laminar boundary layer at sensor TF03. Representative intermittency results are shown in figure 15 comparing results without a shock generator and cases with shock generator angles of 2 and $4^{\circ}$ with nominal shock impingement at $x=400 \mathrm{~mm}\left(R e_{1}=14.2 \times 10^{6} \mathrm{~m}^{-1}\right)$. The figure includes fits to the Narasimha equation. Earlier and more rapid transition 


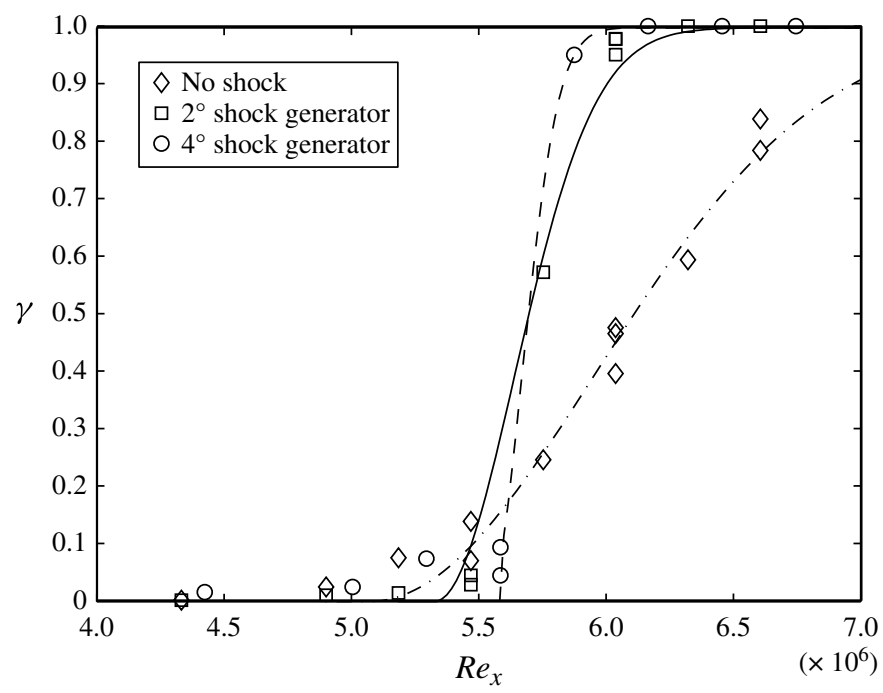

FIGURE 15. Educed intermittency distribution from HEG tests for cases with no shock and cases with shock generator angles of 2 and $4^{\circ}$, showing the increasingly rapid transition process. The no shock and $2^{\circ}$ cases were at $R e_{1}=14.2 \times 10^{6} \mathrm{~m}^{-1}$ and the $4^{\circ}$ case was at $R e_{1}=14.5 \times 10^{6} \mathrm{~m}^{-1}$. In each case the nominal inviscid shock impingement was at $x=0.4 \mathrm{~m}$. The lines are fits to (4.1).

is obtained when the shock is in place and for the $4^{\circ}$ case the transition occurs in between two measurement points (sensors TF08A/B and TF09).

DNS provides access to complete flowfields so in principle many measures of intermittency can be defined. Here we confine ourselves to measures based on the wall $S t$, i.e. to what can in principle be measured in experiments. The full p.d.f.s are accessible but for the flow conditions used these did not show clear separation into two peaks for laminar and turbulent flow regions. Nor were turbulent spots well developed in visualisations of the flow. Several different methods were tested to extract a measure of the intermittency, including the following for which results will be presented. The first method (A) is based on the value of $S t / S t_{l f p}$, where $S t_{l f p}$ is the laminar flat plate solution without shock wave. In this method the DNS flowfield is first filtered with a physical space top-hat filter extending over $3 \delta_{0}^{*}$ in the streamwise and spanwise directions. In wall units for the turbulent boundary layer just downstream of transition this is equivalent to a filter size of $180 \times 180$ which is large enough to filter out flow features associated with the viscous sublayer. A threshold of $S t / S t_{l f p}=2$ was used. In the second method (B) the same filtering is used as in (A), but the detection is based on $\left(S t-S t_{l f p}\right) \mathrm{d} S t / \mathrm{d} z$. The inclusion of a derivative in the spanwise direction ensures that this definition return a value of zero intermittency in purely laminar flow, even with shock interaction. The subtraction of the laminar flat plate solution is included to avoid high intermittency levels when external disturbances are present in the pretransitional flow. In the third method (C) no filtering is applied, but instead a p.d.f. is generated of $\left(S t-S t_{l f p}\right) \mathrm{d} S t / \mathrm{d} z$ within a $3 \delta_{0}^{*} \times 3 \delta_{0}^{*}$ subset of the domain centred at the required point. Then the inter-quartile range is computed and a threshold applied to determine the intermittency. This definition was chosen to mimic the inter-quartile method used in H2K. In the final method (D) the same filtering is used as in A, but the threshold is applied to $S t / S t_{\text {lam }}$, 


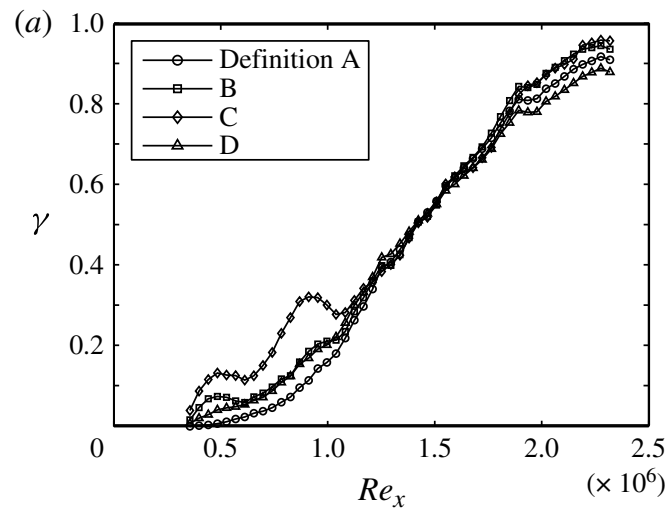

(b)

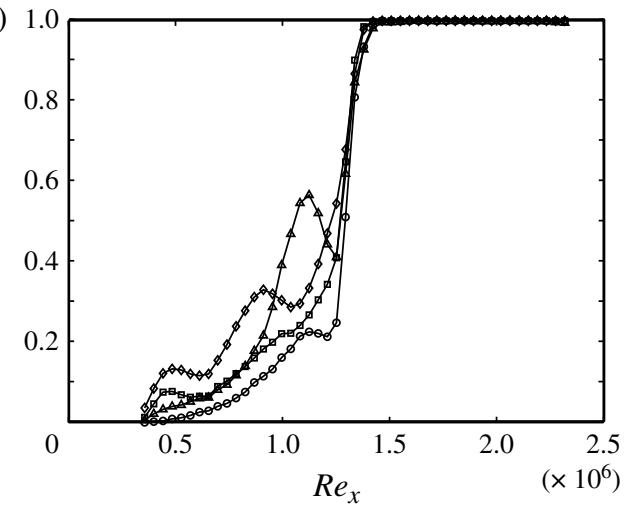

FIGURE 16. Comparison of four different intermittency detection schemes using DNS data: (a) without shock, case NS-H, $(b)$ with shock, case S-H. A: threshold on $S t / S t_{l f p}$, B: threshold on $\left(S t-S t_{l f p}\right) \mathrm{d} S t / \mathrm{d} z$, C: interquartile range on $\left(S t-S t_{l f p}\right) \mathrm{d} S t / \mathrm{d} z$, D: threshold on $S t / S t_{\text {lam }}$.

where $S t_{\text {lam }}$ is the 2D laminar DNS solution, including SWBLI where present. This definition guarantees zero intermittency for cases with laminar interaction.

To facilitate a comparison, the thresholds for methods B-D were chosen so that the intermittency curves were aligned at $\gamma=0.5$ for the no-shock case. It should be noted that, with all of the definitions, the intermittency curves are sensitive to the particular threshold, shifting upstream for lower threshold values and downstream for high values. For example, with method A, varying the threshold between 1.5 and 2.5 led to a change in $\operatorname{Re}_{x_{T}}$ of approximately $\pm 0.2 \times 10^{6}$. A comparison of the four methods is given in figure 16 for simulations $(a)$ without (case NS-H) and $(b)$ with a shock wave (case $\mathrm{S}-\mathrm{H}$ ). In the case with no shock, the curve shapes are similar and generally follow the form expected. Method $\mathrm{C}$ shows large variations upstream of transition, possibly due to the limited sample size used. The method worked well in the $\mathrm{H} 2 \mathrm{~K}$ experiment where long run times resulted in larger datasets. Method A gives a distribution that follows closely the Narasimha (1985) (4.1). However this definition by itself is of limited use as it gives non-zero values for $\gamma$ in strong laminar interactions. Method D fixes this problem, but both methods $\mathrm{A}$ and $\mathrm{D}$ show unusual behaviour for cases with shock impingement, as shown in figure $16(b)$ where it can be seen that there are under- or overshoots in $\gamma$ in the early stages of the shock interaction. In particular, it can be seen that $\gamma$ reduces in the early stages of the interaction, caused by the reduction in the mean St. This seems non-physical, or at least not such a useful definition of $\gamma$, and nor is method D which exhibits a strong overshoot in this region, due to the large separation present in the laminar reference solution used in the definition of $\gamma$. The most useful definition appears to be method B. Some pretransitional oscillations are present, at a reduced amplitude compared with method $\mathrm{C}$, but only a very small undershoot is present before the interaction. We therefore use definition B to compare all the cases with shock impingement.

The intermittency distribution is shown in figure 17(a), comparing the three high-amplitude cases with different shock impingement points with a case with no shock. It can be seen that the intermittency curves follow the no-shock case up to a certain location and then peel off, showing a steep rise thereafter up to fully turbulent conditions $\gamma=1$. The computed intermittency distributions for cases 

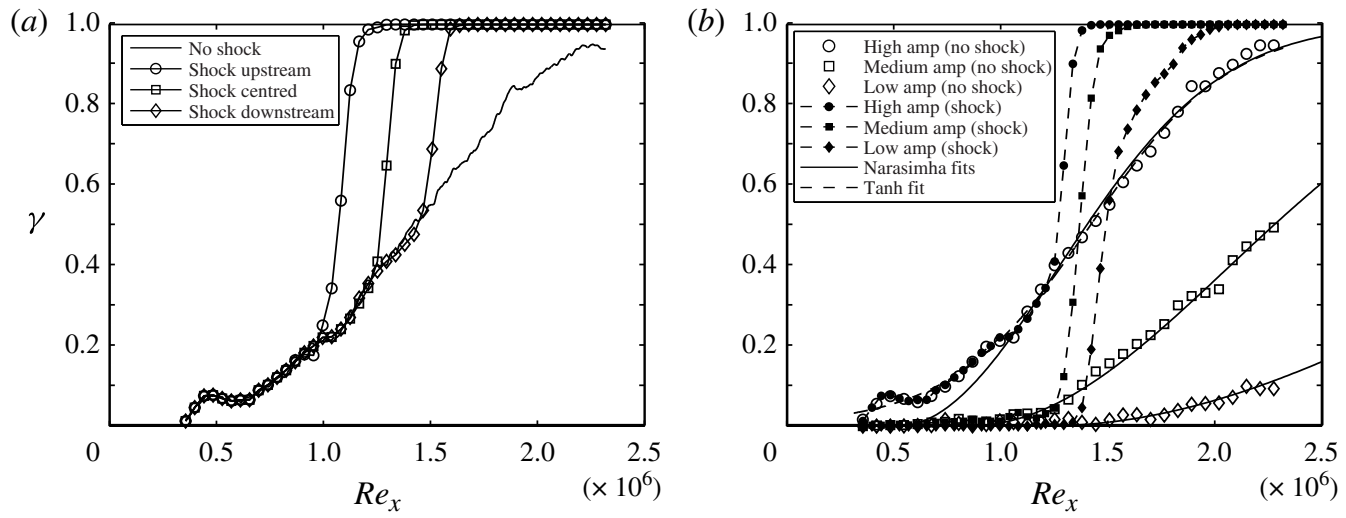

FIGURE 17. DNS intermittency distributions showing (a) effect of shock impingement location (cases S-HU, S-H, S-HD and NS-H) and (b) effect of disturbance amplitude (cases S-H, S-M, S-L with shock and cases NS-H, NS-M, NS-L without shock), with fits to the Narasimha distribution (4.1) and, for cases $\mathrm{S}-\mathrm{H}$, also a fit to a hyperbolic tangent distribution.

with and without shock impingement and three different amplitudes are plotted in figure 17(b). The reduced amplitude cases without shock impingement do not undergo complete transition within the computational domain, reaching intermittency levels of $50 \%$ for the medium-amplitude case and $10 \%$ for the low-amplitude case, compared with the $95 \%$ achieved for the high-disturbance-amplitude case. By contrast all cases with shock impingement undergo a transition process that is completed within the domain. It is interesting that with each halving of disturbance amplitude there is only a small rearward shift of the intermittency curve, of approximately $\Delta\left(R e_{x}\right)=100000$. The much more rapid transition process is suggestive of a change from a mechanism involving the slow growth and merging of spots (although these are not very well distinguished in the visualisations that follow) to a much more rapid (possibly exponential) process based on the breakdown of high-shear layers formed by the shock impingement. Figure 17(b) includes fits to the Narasimha formula 4.1 and also to a hyperbolic tangent. As with the H2K data shown earlier the hyperbolic tangent fit is better upstream of the interaction for these hypersonic flow conditions.

A comparison of transition onset Reynolds numbers and transition duration Reynolds number according to the Narasimha (1985) model (4.1) is shown in table 2. Before discussing the results in detail we should note the limitations on the experimental data due to the small number of measurement locations in the transition zone and the sensitivity of the results in the $\mathrm{H} 2 \mathrm{~K}$ results to the threshold levels $S_{p}$ and $S_{q}$ (up to $30 \%$ variation in individual data points for a $50 \%$ change in threshold). We have already seen the sensitivity of the DNS to the method used to extract this quantity. Nevertheless there are some identifiable trends in the table. First it is noted that the cold wall case (HEG) has a much higher $R e_{x_{T}}$, due to the finite leading-edge radius that was applied to move the transition location downstream onto the instrumented test plate. The sensitivity to the leading-edge geometry (also taking into account a change in properties at the edge of the boundary layer compared with the free stream) may explain some of the variation in $R e_{x_{T}}$, but a rising trend is seen in other measures of the transition progress (for example, the $\gamma=0.5$ location), consistent with the RWG observations of higher transition Reynolds numbers at 


$\begin{array}{lcccc}\text { Case } & \operatorname{Re}_{1}\left(\mathrm{~m}^{-1}, \text { experiment) }\right. & \text { Amplitude (DNS) } & \operatorname{Re}_{x_{T}} & R e_{\lambda} \\ \text { H2K } & 3.3-11.7 \times 10^{6} & - & 0.70 \times 10^{6} & 0.75 \times 10^{6} \\ \text { HEG } & 14.2 \times 10^{6} & - & 5.1 \times 10^{6} & 0.88 \times 10^{6} \\ \text { DNS } & - & \text { High (NS-H) } & 0.52 \times 10^{6} & 0.68 \times 10^{6} \\ \text { DNS } & - & \text { Medium (NS-M) } & 0.85 \times 10^{6} & 1.01 \times 10^{6} \\ \text { DNS } & - & \text { Low (NS-L) } & 1.20 \times 10^{6} & 2.0 \times 10^{6}\end{array}$

TABLE 2. Collected results from fits to the Narasimha (1985) intermittency formula (4.1) for cases without shock interaction. The transition onset Reynolds number is denoted with $R e_{x_{T}}$ and the transition duration with $R e_{\lambda}$.

higher unit Reynolds numbers. The DNS results show increasing $R e_{x_{T}}$ with decreasing disturbance amplitude, which is obvious. The DNS also show an increasing trend in $R e_{\lambda}$ with reducing disturbance amplitude, which in the Narasimha (1985) model would be explained as a reduction in the spot formation rate $n$, since in the model $\lambda$ is inversely proportional to $n \sigma$ where the spot growth rate $\sigma$ would not be expected to be affected by the disturbance amplitude. The medium- and high-amplitude DNS cases show values of $R e_{\lambda}$ that are consistent with all of the experiments. It is interesting that even the HEG experiments show the same values, implying, for the same spot creation rate $n$, that the spot growth rate $\sigma$ is not strongly affected by the cold wall temperature. In a DNS study of isolated turbulent spots Redford et al. (2012) showed a small reduction in spot growth rate with decreasing wall temperature, but this was a secondary effect to that of Mach number. It is also worth noting that the relation $R e_{\lambda}=10 R e_{x_{T}}$ given in Narasimha (1985) does not hold for the cases shown here. Clearly the spot growth rate is different in compressible flows (a factor of four lower than in incompressible flow), but also the spot creation rate may be different due to the stronger receptivity of high-speed boundary layers to acoustic disturbances compared with vortical disturbances.

Alongside the computed intermittency distributions is it useful to look at instantaneous visualisations. Figure 18 shows slices through the streamwise momentum field $\rho u$ in a plane parallel to the wall. The particular plane chosen is at $\hat{y}=0.3208$ based on wall scaling for the turbulent region in a case without a shock wave. For comparison, the first frame shows the medium-amplitude case with no shock, indicating an extended transition region without well-developed turbulent spots, but with an emerging wedge of turbulent fluid occurring alongside pretransitional flow with large streaky structures. By contrast the lower three frames, all with shock impingement, show a rapid breakdown to turbulence consistent with the intermittency results. The resulting turbulent flow contains smaller scale and more intense features, as expected from the large overshoot in skin friction seen in figure 9. In the low-amplitude case the transition process is incomplete and the post-interaction flowfield show wedges of turbulent fluid alongside streaky pretransitional flow. In the low- and medium-amplitude cases it can be observed that the growth angle of the main turbulent wedge increases during the interaction, which was also observed in earlier work of Krishnan \& Sandham (2007) where individual turbulent spots were studied in the context of a shock-induced separation bubble and enhanced spot growth rates of up to a factor of four were observed. 

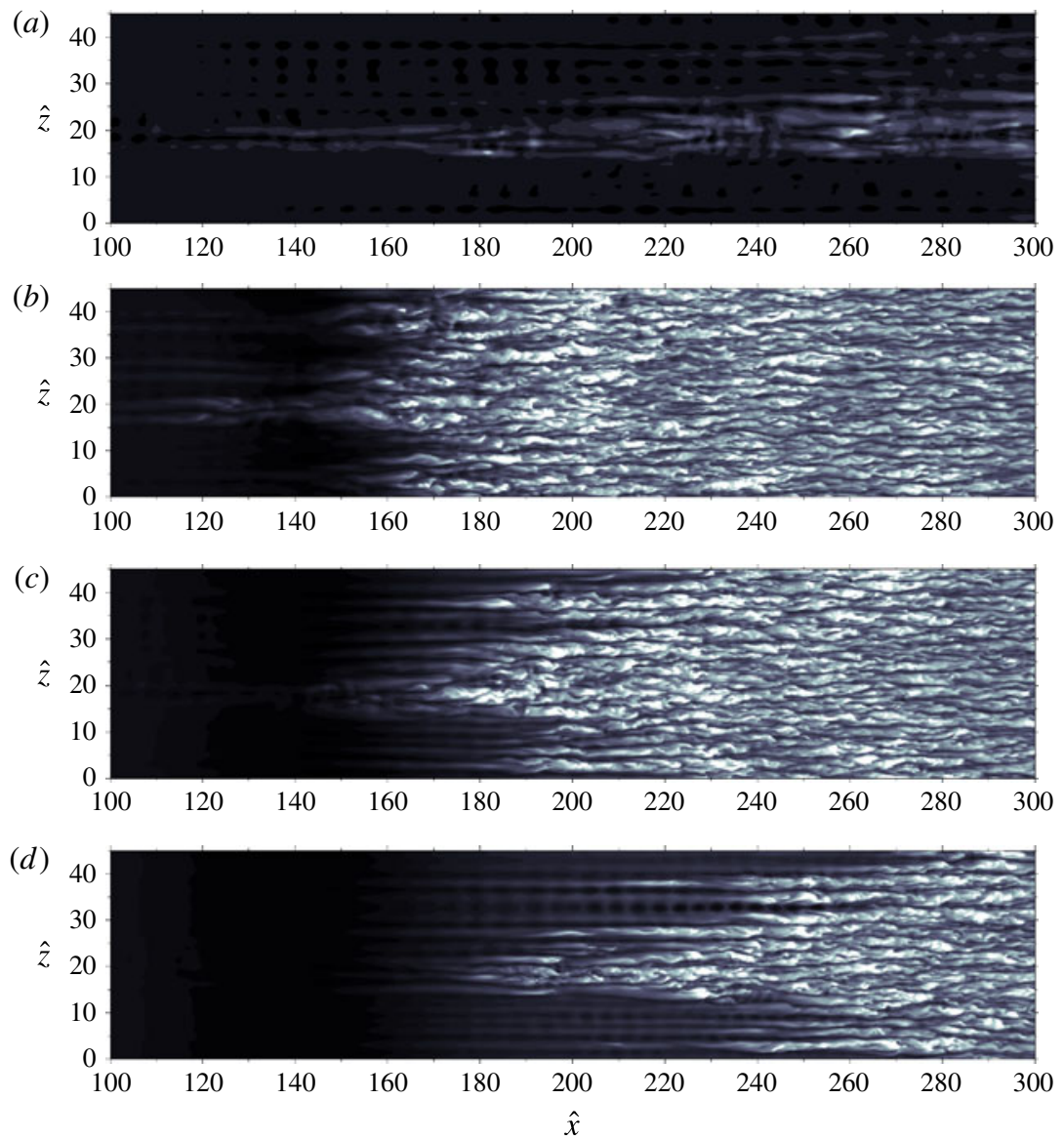

FIgURE 18. (Colour online) Contours of $\rho u$ at a fixed plane $\hat{y}=0.3208$ for $(a)$ medium amplitude with no shock (case NS-M), compared with cases with shock $(b)$ high amplitude (case $\mathrm{S}-\mathrm{H}$ ), (c) medium amplitude (case $\mathrm{S}-\mathrm{M}$ ) and $(d)$ low amplitude (case S-L).

\section{Mechanisms of transition: second (Mack) modes, streaks and spots}

The detection of second (Mack) modes in hypersonic boundary layers is challenging and much of the experimental data is for cone flows rather than flat plates. The presence or absence of second (Mack) mode instabilities could not be confirmed from PCB measurements in HEG due to the short test times, but results from RWG and $\mathrm{H} 2 \mathrm{~K}$ suggested that the modes were present. In both cases PCB sensors were used. In RWG a Wiener filter had to be applied to the data to improve the signal-to-noise ratio. A dominant mode was observed at $f=200 \mathrm{kHz}$ at PCB1. The Mack mode frequency can be estimated as $f=U_{e} /(2 \delta)$, where the $99 \%$ laminar boundary-layer thickness $\delta$ at the experimental run conditions is given by $\delta=12 x R e_{x}^{-1 / 2}$. This gives an estimate of $f=199 \mathrm{~Hz}$ which is in close agreement with the experimental measurement. This peak shifted to lower frequency $(160 \mathrm{kHz})$ at sensor PCB3 from the same run, consistent with the thickening boundary layer downstream. For the same sensor, PCB1, for different unit Reynolds numbers it was found that the frequency increased 

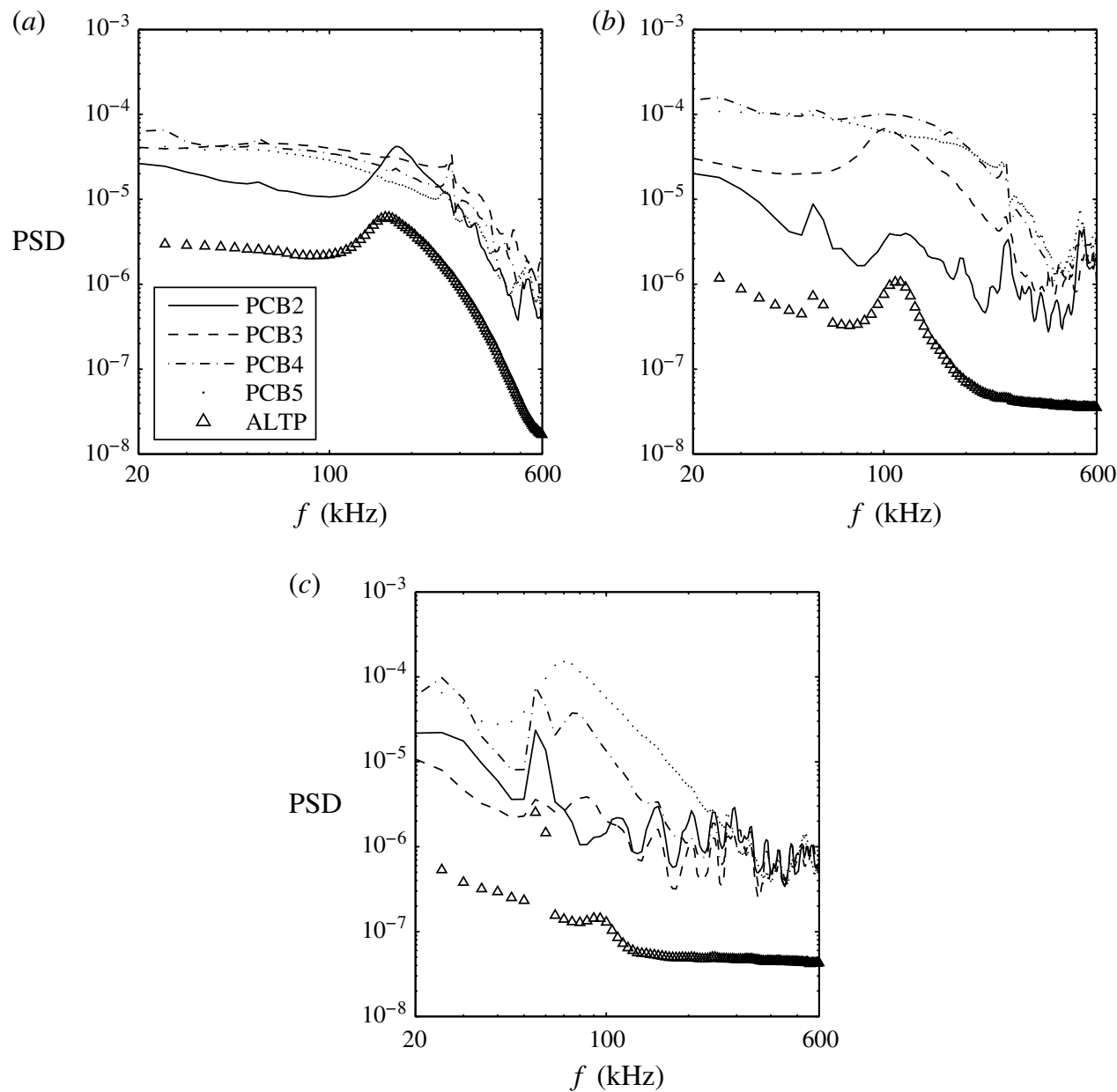

FIGURE 19. H2K spectra at different unit Reynolds numbers $(a) R e_{1}=11.65 \times 10^{6} \mathrm{~m}^{-1}$, (b) $R e_{1}=6.17 \times 10^{6} \mathrm{~m}^{-1}$ and (c) $R e_{1}=3.30 \times 10^{6} \mathrm{~m}^{-1}$. Pressure data was obtained from PCB sensors 2 to 5 . The ALTP data have been multiplied by a factor of $10^{4}$ so that the PSDs can be compared on the same scale.

to $f=233 \mathrm{kHz}$, which is consistent with the boundary layer thinning at a fixed $x$ location as $R e_{1}$ increases.

Additional measurements confirming the presence of second (Mack) modes were obtained in $\mathrm{H} 2 \mathrm{~K}$ using PCB and ALTP sensors. These results were taken from a $5 \mathrm{~s}$ window during the steady flow phase. A Welch method was used with 1000 blocks with $50 \%$ overlap; no additional filtering was applied. Figure 19 shows the power spectral density (PSD) of pressure at three values of $R e_{1}$, measured by PCB sensors PCB2-PCB5 at increasing streamwise locations as shown in figure 2. The figure also shows the PSD measured by an ALTP sensor that was located at the same streamwise position as PCB2. For this sensor the PSD of the Stanton number was plotted scaled with a factor of $10^{4}$. The raw PCB signals show interference effects and are increasingly noisy at frequencies above $200 \mathrm{kHz}$. The ALTP sensor PSD delivered much smoother spectra, although the same interference at $60 \mathrm{kHz}$ is again visible. Second (Mack) modes are clearly visible and consistent between the ALTP and PCB 

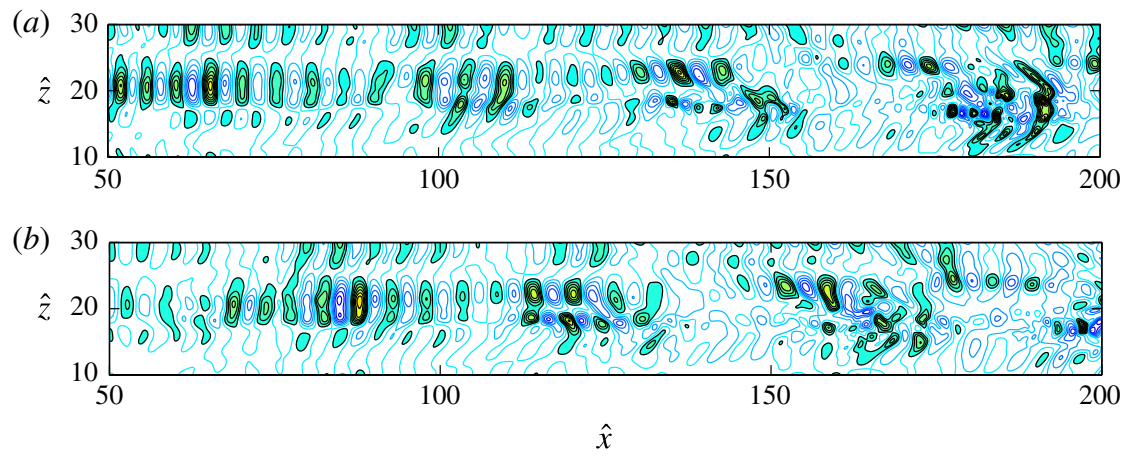

FIgure 20. (Colour online) Contours of wall pressure (using filled contours for pressure below the free-stream pressure) from DNS for case NS-M. Contours are shown at two time instants, $(a)$ and $(b)$, separated by half a period of the lowest forcing frequency.

sensors, for example the peak around $180 \mathrm{kHz}$ in figure $19(a)\left(R e_{1}=11.65 \times 10^{6} \mathrm{~m}^{-1}\right)$ and the peak at $110 \mathrm{kHz}$ in figure $19(b)\left(R e_{1}=6.17 \times 10^{6} \mathrm{~m}^{-1}\right)$. The decrease in frequency with decreasing $R e_{1}$ is consistent with thicker boundary layers at the lower unit Reynolds numbers. From figure $19(b)$ it can be seen that the second (Mack) mode grows rapidly from sensor PCB2 to PCB3 and shifts to lower frequency, consistent with an unstable Mack mode and a thickening boundary layer downstream. A similar trend can be seen in figure 19(c) from PCB3 to PCB5, with an overall growth of nearly two orders of magnitude in the PSD. Second (Mack) modes were also found with shock interaction, for shock impingement at 239 and $331 \mathrm{~mm}$. In this case the frequency was seen to shift to lower frequencies in the early stages of the interaction, for example from 120 to $50 \mathrm{kHz}$ for the ALTP sensor at the nominal $R e_{1}=11.65 \times$ $10^{6} \mathrm{~m}^{-1}$ flow condition and shock impingement at $229 \mathrm{~mm}$. This is again explained by the rapid thickening of the boundary layer in the early stages of the interaction.

The DNS data can be used to look in more detail at the origins of transition. Figure 20 shows a slice through the wall pressure at one instant in time, illustrating how structures with a streamwise wavelength of the order of 5-10 $\delta_{0}^{*}$ occur in distinct wavepackets. One such wavepacket can be seen to be breaking down near $\hat{x}=180, \hat{z}=20$. A characteristic feature is the spanwise dislocations that occur between the wavepackets. Some spanwise variation was expected since the inflow forcing did not include two-dimensional waves. From investigations of instantaneous datasets, it was found that the initial spanwise dislocations are correlated with low-speed streaks near the wall, while the strongest streak emerges at $\hat{z}=20$ during the breakdown stage. No evidence is seen of subharmonic breakdown. Instead, the wavelength of the breakdown is consistent with a fundamental secondary instability. The final breakdown also shows elements of a sinuous streak instability, for example near $\hat{x}=125$ in figure $20(b)$.

We can readily identify the wavepackets as second (Mack) modes. From figure 20 the wavepacket centred at $\hat{x}=105, \hat{z}=20$ can be tracked through two cycles of the lowest frequency of the forcing, up to its breakdown, centred at $\hat{x}=185, \hat{z}=20$. Since two periods of forcing corresponds to a period of 100 (dimensionless) this corresponds to a convection speed in the region of $80 \% U_{e}$ (which is close to the group velocity of the Mack modes $c_{g}=0.82 U_{\infty}$, but also close to the convection speed of slow acoustic disturbances in the free stream $\left.u-c=5 U_{\infty} / 6\right)$. The spatial structure of the modes can be seen more clearly in figure 21 , which shows slices at $\hat{z}=21$ with 

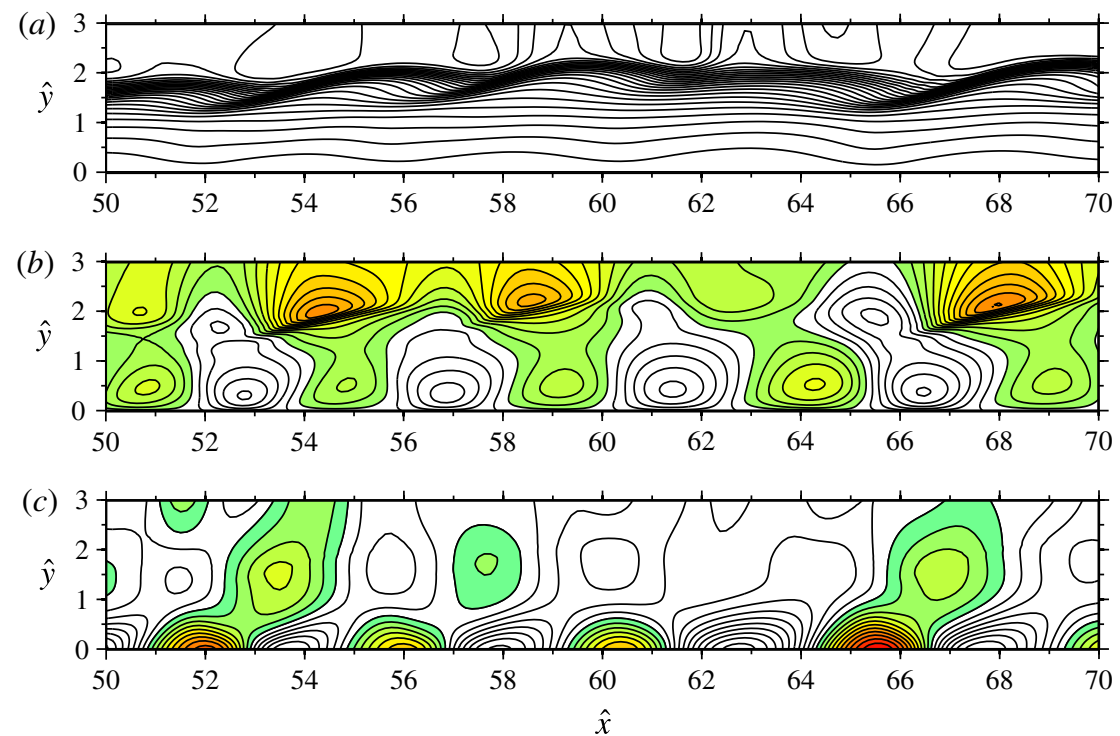

FIgURE 21. (Colour online) Slices at $\hat{z}=21$ showing contours of $(a) \rho u,(b) \rho v$ and (c) $p-p_{\infty}$ illustrating the consistency of the structures with the second (Mack) modes of instability.

contours of streamwise momentum, wall-normal velocity and pressure. Here it can be seen how the wavelength grows from 4.0 to 4.5 over the subdomain while the visual boundary-layer thickness grows from approximately 2.0 to approximately 2.2, i.e. with a wavelength twice the boundary-layer thickness. Thus, phase speed and wavelength are consistent with Mack modes, as is the spatial structure of the instability wave (for example Sandham \& Lüdeke (2009) showed similar contours of pressure and normal velocity for a second (Mack) mode in a Mach 6 boundary layer flow). It can also be noted that the wavelength of 4-4.5 means that there are 32-36 grid points per wavelength, so that these waves are well resolved on the computational grid.

\section{Discussion and conclusions}

A combined numerical and experimental approach has been used to study transitional SWBLI at Mach 6. The experiments used the same flat plate test model in three separate facilities with a range of flow conditions and measurement techniques, while the DNS were run for a range of disturbance amplitudes and shock impingement locations. The main outcome has been a successful cross-validation of experimental techniques and DNS, providing a degree of confidence in the latter to provide data not accessible to experiment. Of the measurement techniques employed, accurate measurements of the mean heat transfer were obtained from QIRT in RWG and H2K, while for the disturbances, meaningful spectra and intermittency distributions were obtained from both PCB and ALTP sensors in the H2K facility. In the HEG facility a better signal-to-noise characteristic meant that it was possible to deduce intermittency distributions using hot film sensors.

Experiments in the RWG facility enabled a sweep across a wide range of unit Reynolds numbers, showing a rising and then falling trend in the peak Stanton number as the unit Reynolds number was increased, also corresponding to an increase in the 
intermittency of the upstream boundary layer covering a range from fully laminar to fully turbulent before the shock interaction. Experiments in the H2K facility allowed long run times and the accumulation of enough data to study intermittency and spectra, revealing the presence of Mack modes of instability. Finally the experiment in HEG allowed the study of a case with an extreme cold wall condition, with p.d.f.s showing the presence of both laminar and turbulent regions of flow suggestive of turbulent spots.

In the corresponding DNS both the inflow disturbance amplitude and shock location were varied and for the high-amplitude disturbances the Stanton numbers compared well with the RWG results at a unit Reynolds number of $4 \times 10^{6}$, while the medium-amplitude compared well with the $\mathrm{H} 2 \mathrm{~K}$ results at a unit Reynolds number of $6 \times 10^{6}$. Some quantitative differences remain, for example the minimum $S t$ in the experiments is lower than the DNS and the peak is broader in the experiments. Nevertheless the maximum St number is within $10 \%$ which is considered an excellent result considering the difficulty of the experiments and simulations, with the peak $S t$ being to some degree sensitive to numerical resolution, while the experiment was sensitive to leading-edge sharpness. The remaining differences are almost certainly due to the different disturbance environment and the omission of the plate leading edge from the DNS. Measuring the disturbance field in wind tunnel experiments remains beyond current instrumentation, however in principle it would be possible to include the plate leading edge in the numerical simulations and also to simulate a more realistic disturbance field by including the sound radiating from turbulent boundary layers on the nozzle walls.

A number of conclusions are possible based on the present study. The highest levels of wall heat transfer were consistently obtained for transitional rather than fully turbulent cases, corresponding to flows that are only intermittently turbulent. Intermittency, where it could be reliably measured, could be fitted to Narasimha form without shock impingement, although an alternative hyperbolic tangent distribution showed better correspondence with the data for low levels of intermittency. Cases with shocks always showed a very rapid increase in intermittency in the interaction zone, indicating that lateral spot growth was increased by the shock interaction, although at the lowest disturbance amplitude used in the DNS it was observed that the intermittency did not necessarily reach unity and some regions of laminar flow survived the interaction. The pre-interaction boundary layer is characterised by second mode instabilities, observed in H2K, RWG and DNS. The DNS also showed streamwise streaks and the formation of early stage spots.

\section{Acknowledgements}

The authors would like to acknowledge financial support for this work under an ESA/TRP project (contract number 22793/09/NL/CP). Computer time for the DNS was provided by the UK Turbulence Consortium under grant EP/G069581/1. The authors would also like to thank Dr N. Murray, Dr K. Hannemann and Dr A. Gülhan for technical discussions and support.

\section{Appendix A. Laminar and turbulent reference solutions}

The computational domain was shown in figure 3 in relation to the physical flat plate. Coordinates $\hat{x}, \hat{y}$ and $\hat{z}$ are used in the computational domain and are made dimensionless with a reference length, which is set as the displacement thickness $\delta_{0}^{*}$ of the boundary layer at the inflow to the computational domain. To compare with 


$\begin{array}{lcccccc}\text { Case } & M_{\infty} & T_{\infty}(\mathrm{K}) & T_{w} / T_{\infty} & \Delta_{s} & f^{\prime \prime}(0) & g^{\prime}(0) \\ \text { RWG/H2K } & 6.0 & 65.0 & 4.5 & 8.624273 & 0.494891 & 1.141575 \\ \text { HEG } & 5.7 & 420.0 & 0.7 & 3.157634 & 0.386863 & 1.997440\end{array}$

TABLE 3. Similarity solution for compressible laminar boundary layer at representative flow conditions for each experiment.

experiments we generally use the Reynolds number based on distance from the leading edge of the flat plate, denoted $R e_{x}$. This is connected to the $\hat{x}$ location within the computational domain by

$$
R e_{x}=R e_{x_{0}}+\hat{x} R e
$$

where $R e$ is the simulation Reynolds number (based on the inflow displacement thickness and free-stream conditions) and $R e_{x_{0}}$ is the Reynolds number based on the distance $x_{0}$ from the leading edge of the flat plate to the computational inflow boundary. This latter Reynolds number can be found from the compressible laminar boundary-layer similarity solution (White 2006) as

$$
R e_{x_{0}}=\frac{1}{2}\left(\frac{R e}{\Delta_{s}}\right)
$$

where the scale factor $\Delta_{s}$ is given by

$$
\Delta_{s}=\int_{0}^{\infty} \frac{\rho_{\infty}}{\rho}\left(1-\frac{\rho u}{\rho_{\infty} U_{\infty}}\right) \mathrm{d} \eta,
$$

with $\eta$ a dimensionless similarity coordinate defined by integration of

$$
\frac{\partial \eta}{\partial y}=\frac{\rho U_{\infty}}{\sqrt{2 \rho_{\infty} U_{\infty} \mu_{\infty} x}} .
$$

The scale factor $\Delta_{s}$ from the similarity solution is shown in table 3 for representative conditions of the various experiments referred to in this report. According to this laminar similarity solution the variation of normalised displacement thickness is given by

$$
\frac{\delta^{*}}{x}=\sqrt{2} \Delta_{s} R e_{x}^{-1 / 2}
$$

The variations of skin friction and Stanton number are then found from

$$
c_{f}=\sqrt{2} f^{\prime \prime}(0) R e_{x}^{-1 / 2} \frac{\mu_{w} \rho_{w}}{\mu_{\infty} \rho_{\infty}}
$$

and

$$
S t=\frac{g^{\prime}(0) R e_{x}^{-1 / 2}}{\sqrt{2} \operatorname{Pr}\left(T_{r}-T_{w}\right)} \frac{\mu_{w} \rho_{w}}{\mu_{\infty} \rho_{\infty}},
$$

with the derivatives of similarity variables $f$ and $g$ at the wall given in table 3 and the recovery factor from (2.5).

For turbulent boundary layers we take as a reference the Van Driest II solution for a turbulent boundary layer (Van Dreist 1956). As seems to be common practice 
(e.g. Cary \& Bertram 1974), we include a recovery factor $r=0.9$ in the formulation, although we note that this was not present in the original paper. The Van Driest equation is given by

$$
c_{f}=\frac{1}{\lambda}\left[\frac{0.242\left(\sin ^{-1} \alpha+\sin ^{-1} \beta\right)}{\log _{10}\left(c_{f} R e_{x}\right)-0.76 \log _{10}\left(T_{w} / T_{\infty}\right)+0.41}\right],
$$

where $\lambda=(\gamma-1) M_{\infty}^{2} r / 2, \alpha=\left(2 a^{2}-b\right) / Q, \beta=b / Q, Q=\sqrt{b^{2}+4 a^{2}}, a=\sqrt{\lambda T_{\infty} / T_{w}}$ and $b=(1+\lambda) T_{\infty} / T_{w}-1$. Equation (A 8) is implicit in $c_{f}$ but converges rapidly with a straightforward iteration starting with a guess for $c_{f}$ in the right-hand side. Stanton numbers are obtained from $c_{f}$ using the standard Reynolds analogy (e.g. White 2006)

$$
S t=\frac{c_{f}}{2 \operatorname{Pr}^{2 / 3}}
$$

\section{REFERENCES}

ARnal, D. \& Delery, J. P. 2004 Laminar-turbulent transition and shock wave/boundary layer interaction. Tech. Rep. RTO-EN-AVT-116. NATO RTO.

BABInSKY, H. \& HARVEY, J. 2011 Shock Wave-Boundary-Layer Interactions. Cambridge University Press, Cambridge.

Benay, R., Chanetz, B., Mangin, B., Vandomme, L. \& Perraud, J. 2006 Shock wave/transitional boundary-layer interactions in hypersonic flow. AIAA J. 44 (6), 1243-1254.

BUR, R. \& ChAnetZ, B. 2009 Experimental study on the PRE-X vehicle focusing on the transitional shock-wave/boundary-layer interactions. Aerosp. Sci. Technol. 13 (7), 393-401.

CAnepa, E., Ubaldi, M. \& ZUNini, P. 2002 Experiences in the application of intermittency detection techniques to hot-film signals in transitional boundary layers. In 16th Symposium on Measuring Techniques in Transonic and Supersonic Flow in Cascades and Turbomachines. Cambridge, UK.

CARY, A. M. \& Bertram, M. H. 1974 Engineering prediction of turbulent skin friction and heat transfer in high-speed flow. NASA TN D-7507.

Cook, W. J. \& Felderman, E. J. 1966 Reduction of data from thin-film heat transfer gages: a concise numerical technique. AIAA J. 4 (3), 561-562.

De Tullio, N. 2013 Receptivity and transtion to turbulence of supersonic boundary layers with surface roughness. PhD thesis, University of Southampton, Southampton, UK.

Delery, J. M. 1985 Shock wave/turbulent boundary layer interaction and its control. Prog. Aerosp. Sci. 22, 209-280.

Dolling, D. S. 2001 Fifty years of shock-wave/boundary-layer interaction research: what next? AIAA J. 39 (8), 1517-1531.

VAN Dreist, E. R. 1956 The problem of aerodynamic heating. Aeronaut. Eng. Rev. 26-41.

DuAN, L. \& MARTIN, M. P. 2011 Direct numerical simulation of hypersonic turbulent boundary layers. Part 4. Effect of high enthalpy. J. Fluid Mech. 684, 25-59.

Fiala, A., Hillier, R., Mallinson, S. G. \& Wijesinghe, H. S. 2006 Heat transfer measurement of turbulent spots in a hypersonic blunt-body boundary layer. J. Fluid Mech. 555, 81-111.

Hakkinen, R. J., Greber, I., Trilling, L. \& Abarbanel, S. S. 1959 The interaction of an oblique shock wave with a laminar boundary layer. Tech. Rep. 2-18-59W. NASA Memo.

Hannemann, K., Martinez Schramm, J. \& Karl, S. 2008 Recent extensions to the high enthalpy shock tunnel Göttingen (HEG). In Proceedings of the 2nd International ARA Days, pp. 2008-10-20-2008-10-23. Arcachon, France.

Hedley, T. B. \& KefFer, J. F. 1974 Turbulent non-turbulent decisions in an intermittent flow. J. Fluid Mech. 64 (JUL24), 625-644.

JACobS, R. G. \& Durbin, P. A. 2001 Simulations of bypass transition. J. Fluid Mech. 428, 185-212. 
KATZER, E. 1989 On the lengthscales of laminar shock/boundary-wayer interaction. J. Fluid Mech. 206, 477-496.

KendALL, J. M.. 1975 Wind-tunnel experiments relating to supersonic and hypersonic boundary-layer transition. AIAA J. 13 (3), 290-299.

Knauss, H., Roediger, T., Bountin, D. A., Smorodsky, B. V., Maslov, A. A. \& Srulijes, J. 2009 Novel sensor for fast heat-flux measurements. J. Spacecr. Rockets 46 (2), 255-265.

Krishnan, L. \& SANDhaM, N. D. 2007 Strong interaction of a turbulent spot with a shock-induced separation bubble. Phys. Fluids 19, 016102.

Langtry, R. B. \& Menter, F. R. 2009 Correlation-based transition modeling for unstructured paralleized computational fluid dynamics codes. AIAA J. 47 (12), 2894-2906.

Laurence, S. J., Wagner, A., Hannemann, K., Wartemann, V., Luedeke, H., Tanno, H. \& IтOH, K. 2012 Time-resolved visualization of instability waves in a hypersonic boundary layer. AIAA J. 50 (1), 243-246.

MACK, L. M. 1984 Boundary layer stability theory. Tech. Rep. 705. AGARD.

NARAsimha, R. 1985 The laminar-turbulent transition zone in the boundary layer. Prog. Aerosp. Sci. 22 (1), 29-80.

Neumann, R. D. 1972 Special topics in hypersonic flow. Tech. Rep. 42. AGARD Lecture Series.

Pagella, A., BABUCKe, A. \& RIST, U. 2004 Two-dimensional numerical investigations of smallamplitude disturbances in a boundary layer at $M a=4.8$ : Compression corner versus impinging shock wave. Phys. Fluids 16 (7), 2272-2281.

PATE, S. R. \& SChUeler, C. J. 1969 Radiated aerodynamic noise effects on boundary-layer transition in supersonic and hypersonic wind tunnels. AIAA J. 7, 450-457.

Piponniau, S., Dussauge, J. P., Debieve, J. F. \& Dupont, P. 2009 A simple model for lowfrequency unsteadiness in shock-induced separation. J. Fluid Mech. 629, 87-108.

Redford, J. A., SAndham, N. D. \& Roberts, G. T. 2012 Numerical simulations of turbulent spots in supersonic boundary layers: effects of Mach number and wall temperature. Prog. Aerosp. Sci. 52 (SI), 67-79.

Renk, K. F., Betz, J., Zeuner, S., Lengfellner, H. \& Prettl, W. 1994 Thermopile effect due to laser-radiation heating in thin-films of high-T-C materials. Physica C 235 (1), 37-40.

Reshotko, E. 1969 Stability theory as a guide to the evaluation of transition data. AIAA J. 7, 1086-1091.

Reshotko, E. 2001 Transient growth: a factor in bypass transition. Phys. Fluids 13 (5), 1067-1075.

RoBINET, J.-CH. 2007 Bifurcations in shock-wave/laminar-boundary-layer interaction: global instability approach. J. Fluid Mech. 579, 85-112.

SANDhAM, N. D. \& LÜDEKE, H. 2009 Numerical study of Mach 6 boundary-layer stabilization by means of a porous surface. AIAA J. 47 (9), 2243-2252.

SAVITZKY, A. \& Golay, M. J. E. 1964 Smoothing and differentiation of data by simplified least squares procedures. Analyt. Chem. 36 (8), 1627-1639.

SCHNEIDER, S. P. 1995 Improved methods for measuring laminar-turbulent intermittency in boundary layers. Exp. Fluids 18, 370-375.

SCHNEIDER, S. P. 2008 Development of hypersonic quiet tunnels. J. Spacecr. Rockets 45 (4), 641-664.

SCHUltz, D. L. \& JONES, T. V. 1973 Heat transfer measurements in short-duration hypersonic facilities. Tech. Rep. 165. AGARD-AG.

SteElant, J. \& Dick, E. 1996 Modelling of bypass transition with conditioned Navier-Stokes equations coupled to an intermittency transport equation. Intl J. Numer. Meth. Fluids 23, 193-220.

Steelant, J. \& Dick, E. 2001 Modeling of laminar-turbulent transition for high freestream turbulence. Trans. ASME J. Fluids Engng 123, 22-30.

Stewartson, K. \& Williams, P. W. 1969 Self-induced separation. Proc. R. Soc. Lond. A 312 (1509), 181-206.

Thompson, K. W. 1987 Time-dependent boundary-conditions for hyperbolic systems. J. Comput. Phys. 68 (1), 1-24.

TOUBER, E. \& SANDHAM, N. D. 2009 Large-eddy simulation of low-frequency unsteadiness in a turbulent shock-induced separation bubble. Theor. Comput. Fluid Dyn. 23, 79-107. 
Touber, E. \& SAndham, N. D. 2011 Low-order stochastic modelling of low-frequency motions in reflected shock-wave/boundary-layer interactions. J. Fluid Mech. 671, 417-465.

Wagner, A., Kuhn, M., Schramm, J. M. \& Hannemann, K. 2013 Experiments on passive hypersonic boundary layer control using ultrasonically absorptive carbon-carbon material with random microstructure. Exp. Fluids 54 (10), 1606.

White, F. M. 2006 Viscous Fluid Flow, 3rd edn. McGraw-Hill.

Willems, S. \& Guelhan, A. 2014 Experiments on the effect of laminar-turbulent transition on the SWBLI in H2K at Mach 6. Exp. Fluids (submitted).

Yao, Y., Krishnan, L., Sandham, N. D. \& Roberts, G. T. 2007 The effect of Mach number on unstable disturbances in shock/boundary-layer interactions. Phys. Fluids 19, 054104.

Yee, H. C., Sandham, N. D. \& DJomehri, M. J. 1999 Low-dissipative high-order shock-capturing methods using characteristic-based filters. J. Comput. Phys. 150 (1), 199-238. 